\title{
Article \\ Prebiotic chemistry in the wild: How geology interferes with the origins of life
}

\author{
Craig Walton ${ }^{1, *(\mathbb{D})}$, Paul Rimmer, ${ }^{1,2}$ (i) Helen Williams, ${ }^{1(\mathbb{D})}$ and Oliver Shorttle ${ }^{1,3}$ \\ 1 Department of Earth Sciences, University of Cambridge, Downing Street, Cambridge CB2 3EQ, UK \\ 2 Cavendish Astrophysics, University of Cambridge, JJ Thomson Avenue, Cambridge CB3 OHE, UK. 2MRC \\ Laboratory of Molecular Biology, Francis Crick Avenue, Cambridge CB2 0QH, UK \\ 3 Institute of Astronomy, University of Cambridge, Madingley Road, Cambridge, CB3 OHA, UK \\ * Correspondence: crw59@cam.ac.uk
}

Received: date; Accepted: date; Published: date

\begin{abstract}
A plausible explanation for the origin of life must satisfy constraints imposed by both organic chemistry and early Earth geochemistry. However, the full scope of geochemical parameter space is rarely considered by either theoretical or experimental models of abiogenesis. Here we propose a novel approach, which can make maximum use of available data. We posit that constructive and destructive geochemical interferences with proposed prebiotic reaction schemes can be used to restrict plausible environmental parameter space for the origin of life. Our approach is demonstrated by exploring parameter space for dehydration reactions. Such reactions are universally important in extant biochemistry and all proposed prebiotic reaction schemes, yet challenging to perform under plausible conditions. We specifically explore a minimal pathway for RNA synthesis (formaldehyde; ribose; ribose phosphate; adenosine monophosphate; RNA). Based on assembled thermodynamic and geochemical constraints, we identify that low water activity is a key constructive interference in prebiotic chemistry. Critically, the manner in which low water activity is achieved can strongly discriminate between different environmental scenarios. Exploring interference chemistry is hence an effective means of discriminating between competing origin of life scenarios.
\end{abstract}

Keywords: Prebiotic chemistry; early Earth; origin of life

\section{Introduction}

Any consensus scenario for the origin of life will emerge as a self-consistent synthesis of geochemical constraints on the nature of the Early Earth and experimental demonstrations of chemical feasibility. Building on decades of research into these separate research strands, we propose a novel approach to assess the plausibility of origin scenarios: interference chemistry.

We define interference chemistry as the interaction between the environment and prebiotic reaction networks. This interaction may be constructive, e.g., the warming of the environment to melt ice and allow aqueous reactions to occur; or it may be destructive, e.g., the continued heating of the environment and eventual thermal degradation of organic products. The 'environment' encompasses the pressure and temperature at which reactions in nature must occur, and also the geochemical context in terms of atmospheric, aqueous and substrate composition.

The aim of the interference chemistry approach is to understand the efficiency of lab-based prebiotic reactions under different natural environment-analogue conditions and, in doing so, find the overlap between successful experiments and reconstructions of prebiotic planetary environments. Ultimately, interference chemistry posits that the challenge of capturing realistic geochemical complexity in experimental reaction schemes can be re-purposed as a tool with which to discriminate between origin scenarios in terms of their relative plausibility.

Here, we take some first steps towards this goal, identify shortfalls in the present scalability of the approach, and propose several possible research avenues that would resolve such issues. We do not attempt to identify an exact scenario for the origin of life. However, we do demonstrate the utility of interference chemistry as a tool for sweeping pre-biogeochemical parameter space. 


\subsection{Prebiotic chemistry: a parameter space problem}

Measuring progress in origin science

The last decade has seen a revolution in the field of origins chemistry. Potentially prebiotic syntheses have now been published for membranes, information-storing polymers, and compounds that appear universally in extant metabolism [1,2]. Despite this progress, substantial problems remain with linking these promising chemical pathways into a coherent and prebiotically plausible scenario for the origin of life.

Successful syntheses demand a level of chemical selectivity that is generally absent in realistic geochemical scenarios. Meanwhile, the unavoidable complexity of geochemical environments has yet to be incorporated effectively into most experimental reaction schemes, which calls into question their applicability to realistic early Earth conditions. These long-noted problems (e.g., [3,4]) are highlighted by an ongoing proliferation in the number of proposed origin scenarios (e.g., crater lakes, hot springs, shorelines, surface, shallow, and deep marine, hydrothermal vents, shallow and deep crustal hydrothermal settings, and so on; Figure 1), which together offer up a continually expanding physical-chemical parameter space in need of rigorous assessment (Figure 1). As a consequence of these problems, the question of how to measure progress in prebiotic chemistry remains as unclear as ever e.g., [5-8].
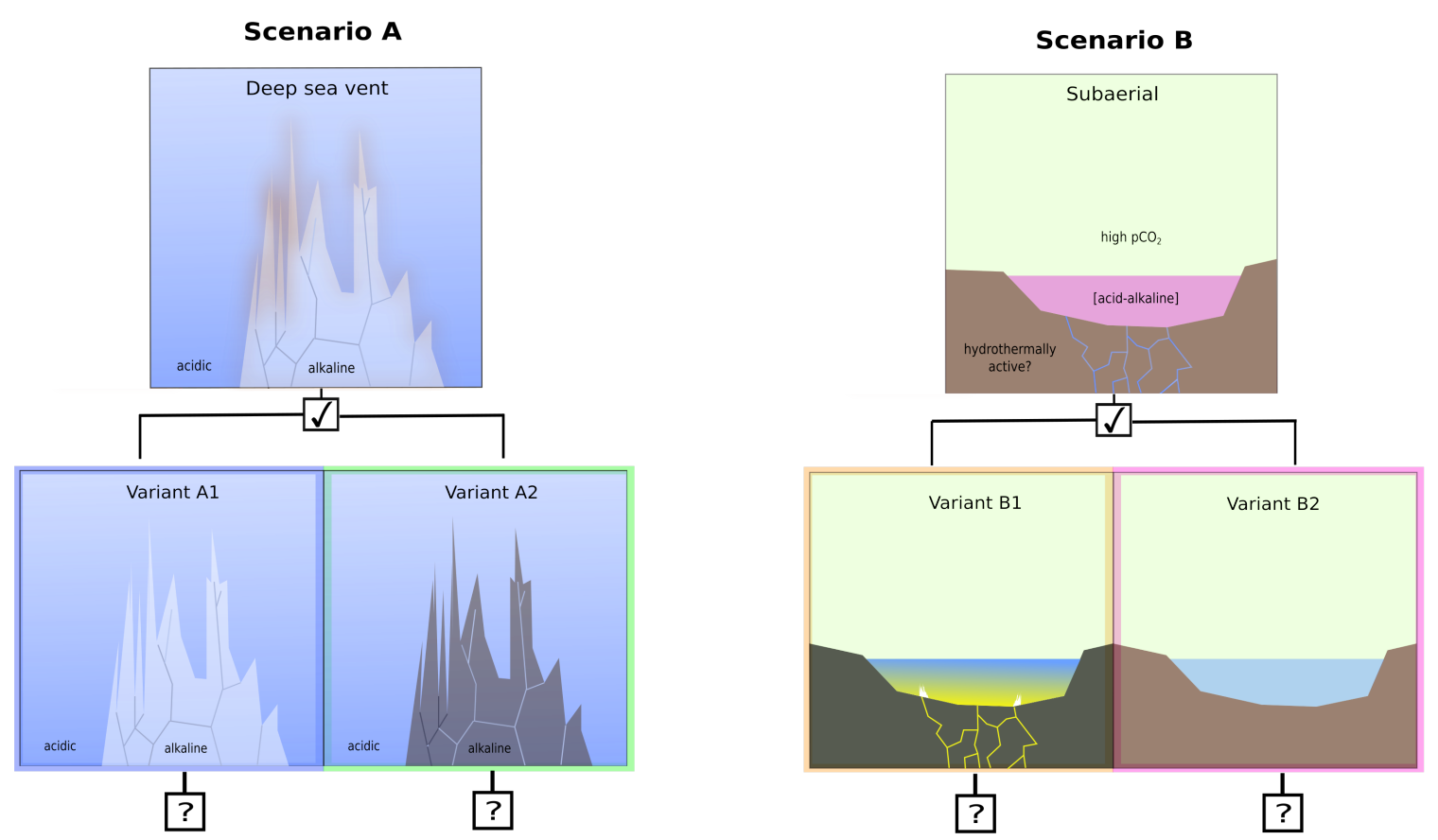

Figure 1. Proliferation of origin scenarios as experimental data is amassed. Whilst high-level scenarios may represent promising and flexible settings for the origin of life, important questions remain about which specific local variants are ideal for promoting prebiotic chemistry. Indeed, the detailed geochemistry of some such variants may prove prohibitive, neutral, or exceptionally advantageous for particular reaction schemes. For this reason, the detailed geochemistry of local plausible environments is important to capture in prebiotic reaction schemes.

\section{Prebiotic systems chemistry}

The unique challenge of prebiotic chemistry can be effectively summarized by noting that no individual experiment is itself necessarily related to the origin of life. As a consequence, any given 
published synthesis is always an exploration of fundamental chemical parameter space, but not necessarily prebiotic parameter space. However, the intractably large scope of the experimental and prebiotic parameter spaces can in principle be sensibly bounded by some basic assumptions:

Assumption 1: the origin of life took place on Earth

Assumption 2: the broad molecular architecture of modern life is essentially conserved from the Prebiotic Era (i.e., some presently unknown fraction of the Hadean Eon, which spans 4.5 to $4.0 \mathrm{Ga}$ )

Assumption 3: central chemical reactions that universally characterize extant biology were proto-typed in the Prebiotic Era (e.g., at a minimum, RNA polymerisation, phosphate-based metabolism, and amphiphile-based compartmentalisation).

Carrying assumptions 1-3 forward, a reasonable model for the origin of life should identify the overlap between plausible environmental parameter space on the early Earth and constructive reaction conditions for the self-assembly, compartmentalization, encoding, and replication of biomolecules.

Difficulties in this approach centrally arise from uncertainty in deciphering the nature of an evolving Earth, and defining the subset of reactions that formed a bridge from the prebiotic to the biotic Earth. Both challenges are compounded by a lack of preserved evidence. No rocks have yet been identified that date to the Prebiotic Era, and (principally geochemical) models have not yet converged on a unique solution for the prevailing deep and surficial conditions of the early Earth. As such, the geochemical context of plausibly prebiotic chemistry remains deeply uncertain.

This latter point masks a critical issue: that 'prebiotic plausibility' is an ambiguous term without further definition and quantification. Despite thoughtful discussion over many years in the literature $[9,10]$, a consensus on how to define prebiotic plausibility in a quantitatively self-consistent manner has yet to be reached. A quantitative framework for prebiotic plausibility would allow for direct competition between origin scenarios, thus providing a measure by which to assess progress in the field at large.

The need to tackle these issues has recently given rise to the concept of prebiotic systems chemistry [11,12]. Systems chemistry posits that increasingly complex experiments are more analogous to the complex chemistry that would have occurred in the Prebiotic Era. Moreover, the approach posits that including some of that complexity may be required to solve important chemical puzzles, e.g., how to buffer reaction $\mathrm{pH}$ or restrict side reactions.

There are obvious advantages to such an approach, including the identification of unexpected connections and barriers between different 'sub-units' of potentially prebiotic chemistry (Pasek et al. [13]). However, we suggest that our ability to assess systems chemistry networks is often still hindered by the manner in which experimental prebiotic and predicted early Earth chemistry have traditionally been compared.

\section{Interference chemistry}

The systems chemistry approach often offers greater insight than studying single reactions alone. However, proponents of the approach have also identified certain limitations [14].

Firstly, the systems chemistry approach of matching a set of reactions that occur in the lab to plausible conducive settings on the early Earth is a relatively inefficient way to explore parameter space. This approach often lacks systematic information regarding the broader environmental dependencies (e.g., P, T, solution chemistry, etc.) of the reaction pathway. It is therefore often challenging to directly compare the corresponding environmental parameter space of different experimental reaction schemes.

A focus on successful experiments in the systems chemistry approach often also leads to a general process of counting the hits and ignoring the misses. However, experiments that fail because of the temperature, pressure, or composition (etc) of the experimental system are successfully identifying 
destructive interferences with the chemistry, and thereby narrowing down the range of viable natural environments in which that specific chemical scenario could occur.

That being said, the relative simplicity and linearity of the single reaction and systems approaches have so far constituted the only reasonable paths for prebiotic chemistry to pursue. This is so because a full parameter space sweep is impossible, at least given the practicalities of a purely experimental approach. It is only now, with a broad understanding of fundamental and potentially plausible prebiotic chemistry assembled, that we can begin to consider an increasingly multi-faceted approach.

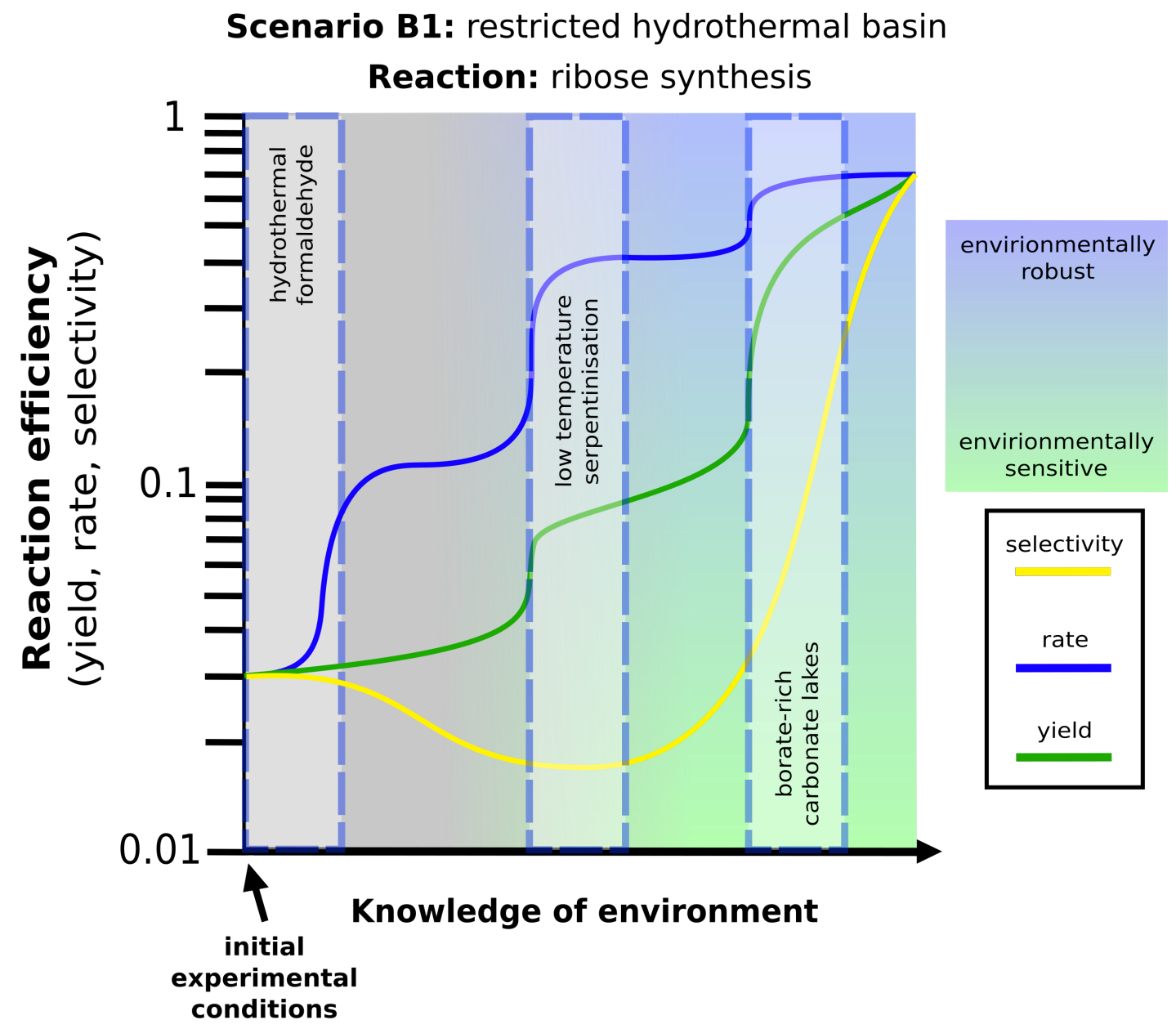

Figure 2. Interference chemistry utilises increasingly realistic reaction conditions in order to identify environmentally robust origin scenarios. Apparent plausibility of the reaction scheme may change in a non-linear manner as new experimental variables are explored.

Figure 2 illustrates our interference chemistry concept. We posit that experiments and quantitative theoretical models which more accurately capture the diversity of variables present in nature should have a greater ability to restrict the plausible parameter space for a given scenario. Measurable aspects of proposed reaction schemes that are potentially sensitive to experimental conditions include yield, rate, and selectivity. Together, these properties represent the efficiency of a given scenario (Figure 2).

We can therefore evaluate the plausibility of a particular origin scenario by considering its efficiency in the face of increasingly realistic interfering environmental conditions, materials, and physicochemical processes.

For example, many chemical species which would have been globally abundant on the early Earth are not generally included in any given prebiotic reaction, due to their apparent irrelevance for the specific chemistry under investigation. Some such species will have little to no effect when 
included, but others will have unexpected constructive or destructive results, e.g., $\mathrm{K}^{+}$[15]. Finally, some will prove unexpectedly transformative (e.g., the incorporation of phosphate in cyanosulfidic reaction schemes [2]) and thus provoke new ways to think about the origin of life.

Interference chemistry as a concept shifts the burden of proof away from obtaining lab-idealised reaction conditions towards describing and reproducing prevailing conditions on the Prebiotic Earth. This would give us a way to efficiently explore the parameter space available to competing origin scenarios and hence measure progress in prebiotic chemistry as a field. In order to demonstrate our approach, we now identify a reasonable reaction pathway for analysis.

\section{Conserved prebiotic reactions}

Proposed synthesis pathways for nucleic acids, proteins, and metabolites are diverse. However, dehydration reactions are fundamentally needed to forge each of these key subsystems of life (Figure 3).

The polymers that comprise cell walls, proteins, and the genetic code are assembled by step-wise dehydration reactions. Meanwhile, phosphorylation-dehydration reactions are crucial for the genesis of ribonucleotide monomers, phospholipid membranes, and the P-bearing molecules that are pervasive constituents of every extant metabolic pathway [13]. As a consequence of these observations, it has long been recognized that any precursor to biology must also have continuously performed dehydration chemistry [16].

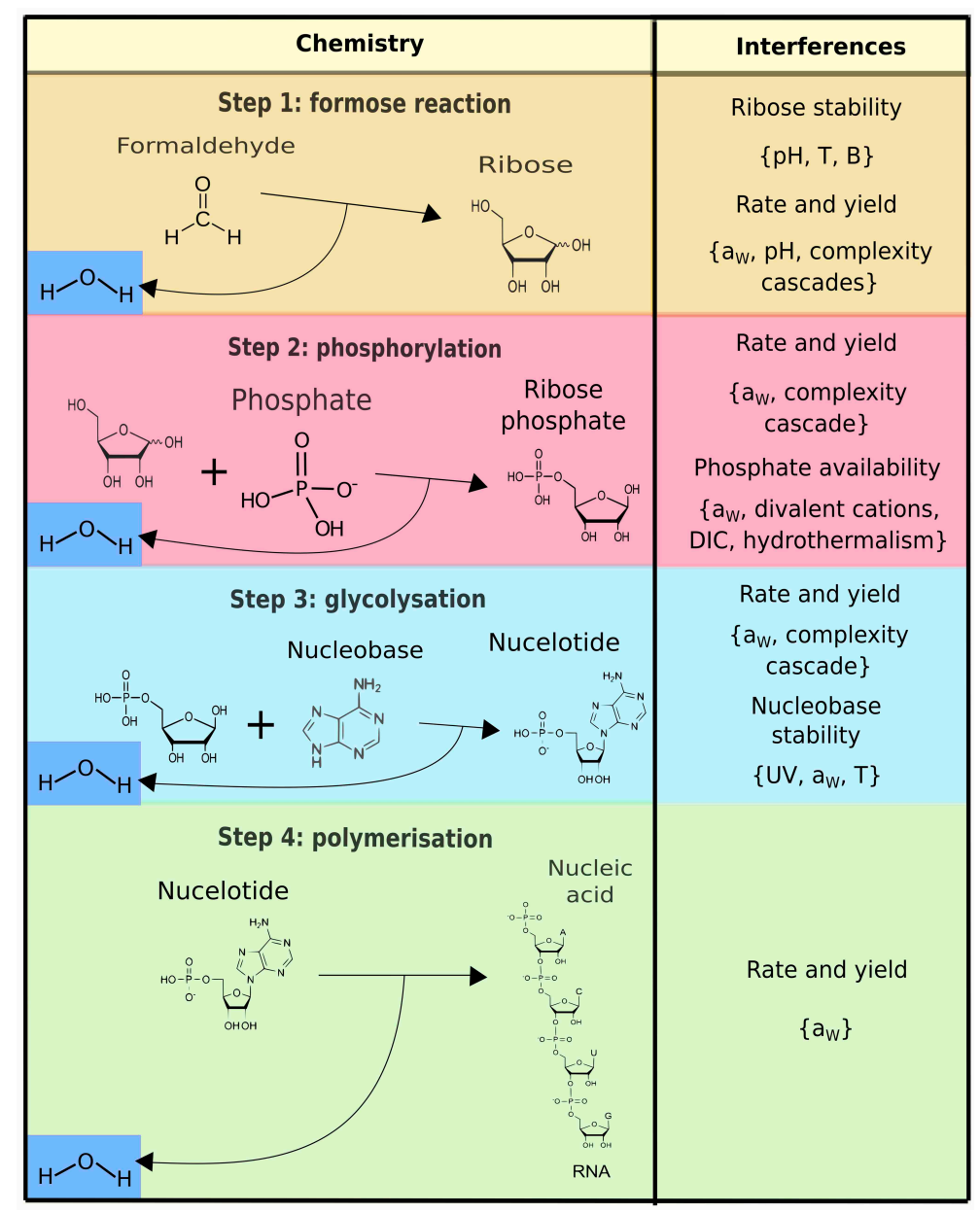

Figure 3. A minimal reaction pathway for RNA synthesis, in which every step constitutes a dehydration reaction. Synthesis of nucleobases is not shown. Key interferences that we consider in this manuscript are indicated. 
However, this point also speaks to a surprising contradiction: despite the striking universality of simultaneously-performed dehydration and hydrolysis chemistry in biochemistry, dehydration reactions are strongly unfavored in dilute aqueous solution [17]. Hence, even whilst the concept of planetary habitability centres on the presence of surficial liquid water, critical aspects of efficient prebiotic chemistry is often confounded by excess amounts of the very same molecule.

This apparent paradox directly links environmental conditions to prebiotic chemistry, thus making dehydration reactions an ideal candidate with to explore using the interference chemistry concept.

Extant biology overcomes the kinetic and thermodynamic challenge of performing dehydration reactions by utilising enzymes. However, enzymes are themselves constructed via enzyme-driven dehydration reactions. We must ask ourselves: which conditions promoted the earliest dehydration reactions in a sufficiently high-yielding, fast-acting, and selective manner to produce functional bio-molecules?

Water is both a direct participant in dehydration/hydrolysis reactions and, in terms of its activity, highly diagnostic of different environments. Hence, identifying conditions that promote potentially prebiotic dehydration chemistry may serve to constrain plausible environments for the origin of life.

\subsection{Biological versus prebiotic dehydration reactions}

Biological dehydration reactions are pervasively accelerated by enzymatic catalysis. Given that extant enzymatic machinery is encoded for by long-chain polymers that themselves form via enzyme-moderated dehydration reactions, a requirement for efficient non-enzymatic chemistry is expressed at the heart of plausible prebiotic chemistry. We can place some constraints on what such a system of reactions might look like by searching for prebiotic analogues to the conditions made possible by enzymes.

\section{Enzymes are not Ostwald catalysts}

An Ostwald catalyst does not change the position of thermodynamic equilibria [18]: the forward and backward reaction rate are increased equally, owing to stabilization of the reaction transition-state [19] (Figure 4). In essence, catalysts reduce kinetic barriers to reaction, but do not change the overall free energy yield of the reaction [18].

Easing kinetic barriers to dehydration/hydration reactions can lead to hydrolysis rates amplified by up to 20 orders of magnitude [20]. However, at least in dilute aqueous solutions, hydrolysis reactions are thermodynamically favoured, whereas dehydration reactions must work 'uphill' in free energy space (Figure 4). Under this condition, lowered activation energies erase the kinetic barrier to spontaneous forward reaction, but the overall equilibrium will still inherently be positioned to favour reactants [21].

In spite of these thermodynamic barriers, highly efficient dehydration chemistry is performed by life with the aid of enzymes. This fact suggests that enzymatic acceleration of thermodynamically unfavored dehydration reactions cannot be explained alone by (strictly defined) catalysis [18]. 


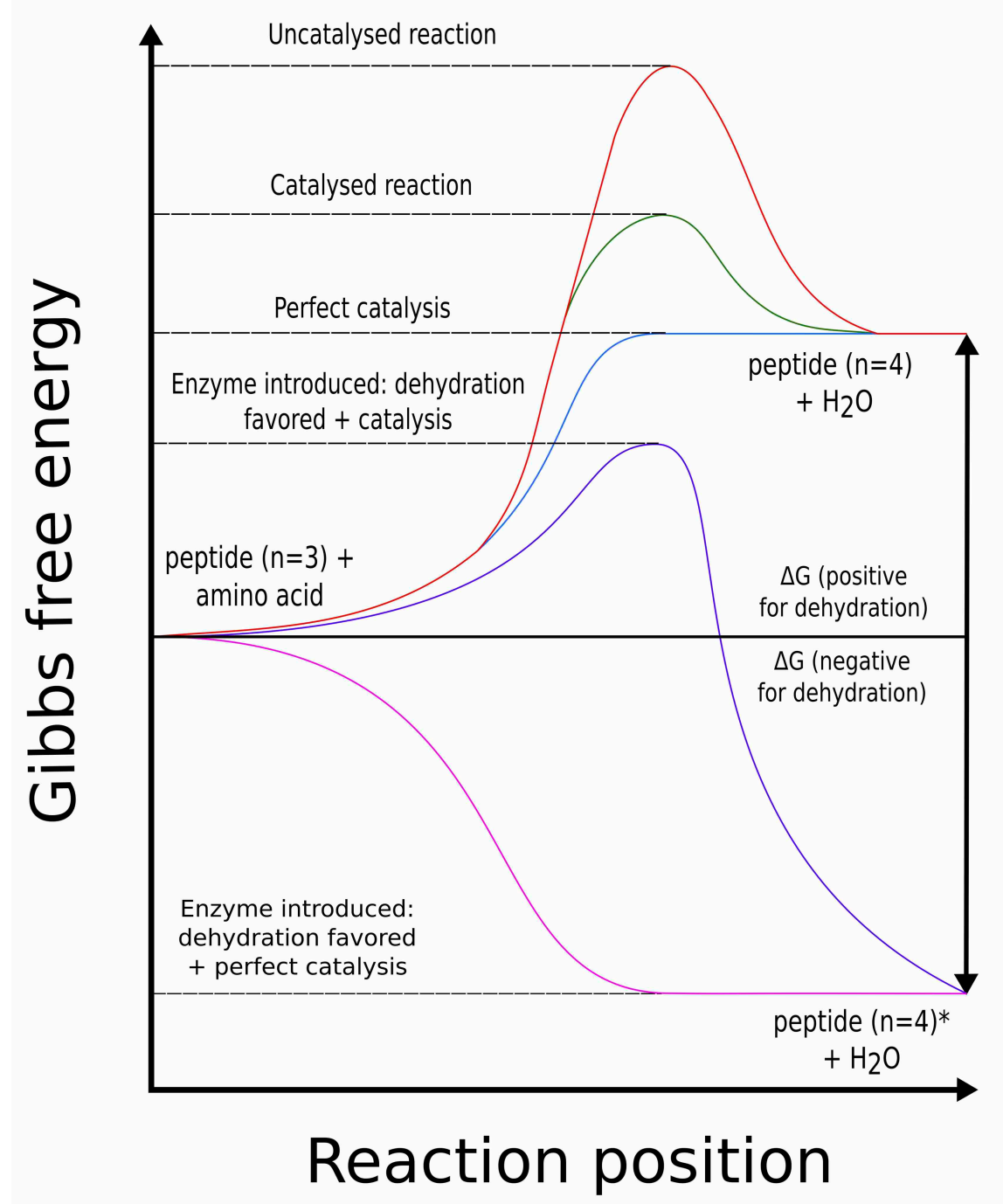

Figure 4. Energy landscapes for a generic hydrolysis/dehydration reaction $A+B \rightarrow C+H 2 O$. Ostwald catalysis is contrasted with a variant of enzymatic catalysis that favours the dehydration reaction. peptide* represents the peptide product stabilised by the enzymatic active cavity.

\section{Enzymes modify water activity}

Biological dehydration reactions are also promoted by enhanced activities of reactants versus the diminished activity of water in the enzymatic active cavity i.e., enzymes are not Ostwald catalysts, as they can modify the thermodynamic landscape and associated equilibrium constants of reactions ([22]; Figure 4).

A general dehydration reaction is

$$
\mathrm{COH}+\mathrm{HX}=\mathrm{CX}+\mathrm{H}_{2} \mathrm{O} \text {. }
$$

At equilibrium, the ratio of products to reactants is expressed as $\mathrm{K}$ - the equilibrium constant.

$$
K=\frac{[\mathrm{CX}] a_{W}}{[\mathrm{COH}][\mathrm{HX}]}
$$

In most treatments of $\mathrm{K}$ for dehydration reactions, $a_{W}$ is not shown in equation [2] owing to its presence in excess (i.e., $a_{W}=1$ ). However, this condition does not hold for low $a_{W}$ systems, where changes in $a_{W}$ will directly favor the dehydration reaction (Le Chatelier's principle). 
Dehydration chemistry may be promoted by the fundamental changes in reaction energy landscapes that take place at water solvated (dilute; $a_{W} \approx 1$ ) vs desolvated (concentrated; $a_{W}<1$ ) conditions. Experimental data for microdroplets confirm that low $a_{W}$ microenvironments in general may promote dehydration as a thermodynamically favored reaction [23].

We therefore see that enzymatic dehydration chemistry is promoted by locally modified ratios and free energies of reactants:products at low water activity. The role of enzymes in stabilising transition states and thereby lowering activation energy barriers (Ostwald catalysis) is clearly important, but only when applied under conditions that thermodynamically favor the product(s) of interest [22].

Given that (1) finding plausible abiotic alternatives to enzymatic catalysis lie at the heart of prebiotic chemistry and (2) water activity is a fundamental environmental condition which enzymes act to perturb, we conclude that the interference chemistry of dehydration reactions is an ideal candidate with which to probe environmental conditions of potential prebiotic interest.

\section{Interference chemistry in RNA synthesis}

We require a toy chemical scenario that is complex enough to illustrate both the utility and present limitations of an interference chemistry approach, yet is simple enough to explore comprehensively.

We select a simple model scenario for RNA formation as our test case: ribose and nucleobase formation, ribose phosphorylation, ribonucleotide formation, and ribonucleotide polymerization. This scenario is chosen for the following reasons: (1) many previous efforts have been made to constrain molecule availability; (2) the availablility of equilibrium reaction constants for each step; (3) the minimal number of steps from basic precursors to RNA, i.e., simplicity and, (4) the high proportion of steps that directly involve removal of water. Figure 3 illustrates the chemistry underlying this particular pathway, in which dehydration reactions feature at every stage: the formose reaction [step 1], phosphorylation [step 2], glycosylation [step 3], and polymerization [step 4].

Evidence has been put forward suggesting the prebiotic plausibility of each step in the pathway shown by Figure 3 [23-25], rendering this simple scheme an ideal candidate on which to apply our suggested methodology of environmental discrimination. However, even the deceptively simple series of reactions shown in Figure 3 has a large number of environment dependencies.

For example, the reaction between adenosine monophosphate (AMP) monomers is directly dependent on the concentrations of AMP (first-order parameter space). However, the plausibility of the reaction is also dependent on the conditions needed to stabilize AMP precursors (second-order parameter space). Linking the interference chemistry of both reaction steps is therefore more informative than considering either step in isolation.

In Section 2, we assess both the environmental parameter space occupied by each species and reaction step shown in Figure 3. We compile available constraints from the literature on molecule availability and determine thermodynamic relationships between reactants and products as a function of water activity in each case. In Section 3, we draw our results together in order to perform an environmental analysis of prebiotic plausibility for our chosen reaction scheme.

\section{Defining and exploring prebiotic parameter space}

We assess the environmental parameter space of each reaction step by considering reaction mechanisms, species availability, and how each environmental condition invoked may constructively and/or destructively interfere with overall reaction efficiency. We consider interferences by water activity, as well as reactant concentrations, complexity cascades (poorly selective forward reactions e.g., the formose reaction, non-directed phosphorylation), UV light, hydrolysis, physical loss (i.e., open vs. closed system reaction conditions), temperature, and $\mathrm{pH}$.

\subsection{Ribose availability}

The first reaction step in Figure 3 is the formation of ribose. 
Ribose availability on the early Earth has been questioned [26] owing to its propensity to self-react via enolization, aldol addition, and retroaldol fragmentation and, secondly, the large number of sugar byproducts produced alongside ribose in most proposed synthesis pathways [27]. Pathways that do not rely on directly producing ribose (e.g., [28]) may avoid these problems. However, for simplicity, we limit ourselves here to the species explicitly shown in Figure 3.

The eponymous formaldehyde of the formose reaction is thought to have been prebiotically abundant, being produced either by photochemical or hydrothermal reduction of $\mathrm{CO}_{2}$ [29]. This provides a plausible stockpile of precursor molecules for ribose formation. However, problems arise when we consider the rapid degradation of ribose (half-life of 1 hour at $100{ }^{\circ} \mathrm{C}$ and $\mathrm{pH}$ 9; [30]) at the very conditions needed to drive the formose reaction in dilute solution: elevated temperature and base-catalysis [7].

Ribose stability can be improved by attaining more moderate temperatures and $\mathrm{pH}$ ( 44 years at 25 ${ }^{\circ} \mathrm{C}$ and $\mathrm{pH} 7 ;$ [30]). Alternatively, the presence of a borate ring moiety improves resistance of ribose to nucleophilic attack e.g., by water; $[27,31]$. Borate further encourages ribose-selective forward reactions [27] i.e, acting as a desirable constructive interference in the reaction scheme.

However, environmental parameter space for borate and/or neutral pH on the Early Earth is restrictive. High concentrations of borate in solution might be achieved via the rehydration of B-rich evaporites or during basin restriction, leading to the formation of B-rich soda lakes. Both evaporites [32] and soda lakes [33] have been proposed to form on the early Earth. However, boron speciation between boric acid $\left(\mathrm{B}(\mathrm{OH})_{3}\right)$ and borate $\left(\mathrm{B}(\mathrm{OH})_{4}^{-}\right)$in aqueous solutions is highly dependent on $\mathrm{pH}$ [34].

For boric acid, $p K_{a}=9.2[34]$ :

$$
\frac{\left[\mathrm{BO}_{4}^{-}\right]}{\left[\mathrm{B}\left(\mathrm{OH}_{3}\right)\right]}=10^{(\mathrm{pH}-9.2)}
$$

Most models of the early atmosphere predict a high $\mathrm{pCO}_{2}$ (greater than 1 bar; [35]), which should produce acidic rainwater and even acidify soda lake settings to $\mathrm{pH} 7$, which can otherwise reach up to pH 11 on the modern Earth [33]. This observation appears to challenge the availability of borate in all but the most extreme end-member lake compositions.

Rain and river water are both thought to have been highly acidic given high $\mathrm{pCO}_{2}$ on the Early Earth (Figure 5; [36]). Even at $\mathrm{pH} \mathrm{7,>99.3 \%} \mathrm{of} \mathrm{B} \mathrm{will} \mathrm{be} \mathrm{speciated} \mathrm{as} \mathrm{boric} \mathrm{acid} \mathrm{in} \mathrm{water,} \mathrm{leaving}$ only $0.7 \%$ as the borate ion. Owing to the high organic concentrations needed for formose-style sugar production, borate is typically used at $>100 \mathrm{mM}$ concentrations to stabilise ribose [27]. While 500 $\mathrm{mM}$ constitutes the highest observed bulk solution boron concentrations on the modern Earth (in closed-basin soda lakes; [33]), achieving similar concentrations of borate on the Early Earth initially appears to be a major challenge for borate-based scenarios to overcome. Possible solutions to this issue may be found by considering the basis of imposed geochemical barriers, in this case atmospheric $\mathrm{pCO}_{2}$, and the interactions with prebiotic organic molecules.

For example, a recent model by Kadoya et al. [37] concluded that impact-induced silicate weathering resulted in low $\mathrm{pCO}_{2}$ during the Hadean Eon. The prebiotic plausibility of borate-rich soda-type basins therefore critically depends on models of early atmosphere chemistry, and establishes a link between rare local environmental conditions and bulk planetary evolution.

However, geochemical constraints alone are insufficient to describe boric acid behaviour in prebiotic environments. Diol-boron interactions in water are well-known to induce changes in the $\mathrm{pKa}$ of boric acid, resulting in a maximum of shift of several log units (to 4-5; Yan et al. [38]). This effect is blunted in phosphate rich environments, where the pkA of boron-diol complexes is maintained at circum-neutral values ([39]). Therefore, even neutral environments, given the presence of sugars, may stabilise ribose-borate complexes. 
However, the formose reaction itself still needs to be driven forwards, which will not happen efficiently without e.g., base catalysis. One option is to exploit the inherent thermodynamic dependence of the formose reaction on water activity.

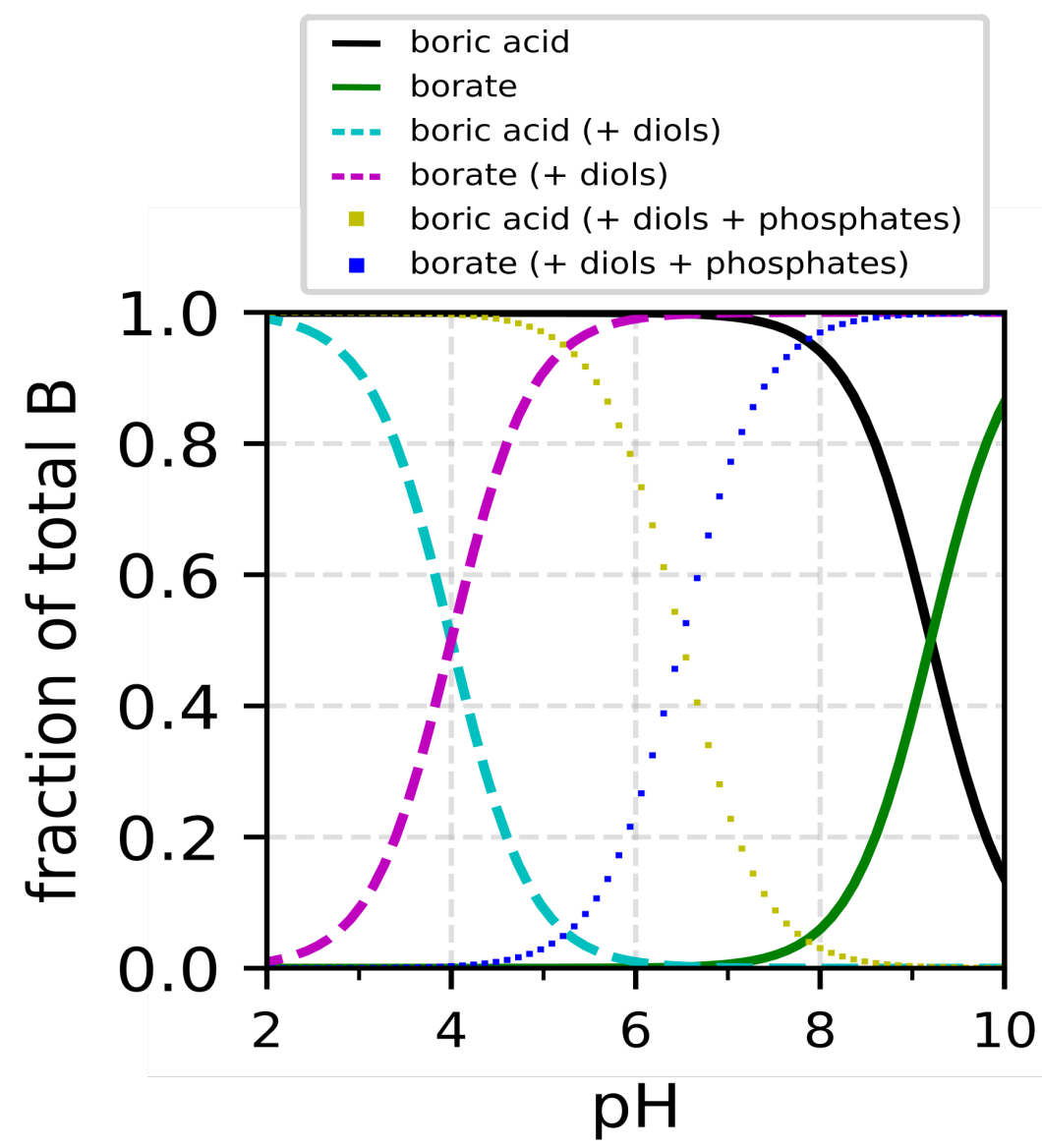

Figure 5. Aqueous speciation of $\mathrm{B}$ as a function of $\mathrm{pH}$ and interfering species. Diol presence $(>10 \mathrm{mM})$ alters the pKa of boric acid. A pKa of 4-5 is achievable with 300-1000 mM diol concentrations. However, the presence of phosphates will result in a pKa of around 6.5, even at these diol concentrations.

Water removal from any given system will force forward (dehydration) reactions to take place within it (moderated by the presence of kinetic barriers). Therefore, low $a_{W}$ reaction conditions may constitute a constructive interference in the formose reaction set. In principle, driving the reaction via low $a_{W}$ would avoid the need for base catalysis in the formose pathway, thereby widening the number of environments in which borate-stabilised sugar synthesis is plausible $[7,30]$.

In the remainder of this section, we use available data to quantify the constructive effects of low $a_{W}$ conditions on phosphorylation, glycosylation, and polymerisation. However, quantitative measurements of equilibrium constants in the formose reaction network are currently lacking, prohibiting a direct prediction of ribose yields as a function of $a_{W}$.

\subsection{Phosphate availability}

Reaction of ribose with dissolved inorganic phosphate (DIP) constitutes the second step in Figure 3. The availability of phosphate in bulk solution will have been governed by the local competition between aqueous geochemical sources, sinks, and recycling pathways.

On the modern Earth, there are few environments in which DIP concentrations approach those often used in prebiotic chemistry (e.g., $>>100 \mu \mathrm{M}$; [33]). Therefore, either a route is required to significantly perturb DIP equilibria on the early Earth, or the geochemical parameter space available 
to origin scenarios is highly restrictive. Figure 6 illustrates DIP concentrations as a function of environmental characteristics and chemistry, which we will now explore.

Lacking biological uptake, $\mathrm{P}$ sinks would have been dominated by phosphate sorption to sinking iron (oxyhydr)oxide particles, the flux of which would have been sensitive to hydrothermal and photolytic oxidation of ferrous iron in the water column [40]. The efficiency of this sink could have been moderated by hydrothermal reflux recycling of phosphite $\left(\mathrm{P}^{3+}\right)$ back into the water column (Figure 6), where phosphate reduction is coupled to iron oxidation at elevated temperature [41]. As phosphite is much more soluble than phosphate, sufficient temperatures achieved during water-rock interaction, combined with some flow back to our putative prebiotic environment, can alleviate the efficiency of the iron-oxide-phosphate flux.

As $\mathrm{P}$ is not a volatile species at the ambient surface conditions estimated for early Earth [42], evaporation combined with a constant finite input flux of P-bearing fluid will simply lead to increasing $\mathrm{P}$ concentrations in any restricted reservoir (Figure $6 \mathrm{~A}$ vs. B). This statement is equally applicable to ocean basins and restricted surface ponds, if on very different scales.

Therefore, wherever the iron-oxide-phosphate flux was sufficiently low, or sufficiently balanced by hydrothermal redox recycling of phosphate in the sediment (Figure 6 C-D), phosphate concentrations in restricted environments could have achieved elevated values relative to the concentrations present in aqueous inputs (e.g., riverine, hydrothermal).

The maximum DIP concentrations achievable in such settings would be set by saturation ceilings for phosphate minerals such as apatite and vivianite, which will have varied strongly as a function of dissolved divalent cation content - ranging from $100 \mu \mathrm{M}$ to $100 \mathrm{mM}$ in Si-rich environments, depending on local $\mathrm{Ca}, \mathrm{Mg}$, $\mathrm{Fe}$, and $\mathrm{CO}_{3}$ concentrations [43].

Trends towards low $\mathrm{Mg}$, $\mathrm{Fe}$, and $\mathrm{Ca}$ concentrations occur during the evaporation of waters that are initially relatively $\mathrm{Cl}$ and $\mathrm{SO}_{4}$ poor but DIC rich. This scenario appears quite reasonable for anoxic pools in equilibrium with a high atmospheric $\mathrm{pCO}_{2}$, but is implausible for saline ocean water [44]. Therefore, prebiotically relevant DIP concentrations are most readily achieved in soda-type surface pond environments (Figure 6).

Exceptions to the above model may also have occurred. Phosphate solubility in alternative solvents has not been quantitatively studied, but a $40 \%$ conversion of hydroxylapatite to highly soluble secondary species (e.g., brushite) was observed in the Urea-Ammonium-Formate-Water (UAFW) solutions experiments of Burcar et al. [45]. Finally, high DIP concentrations may have been achieved on local scales within microenvironments (e.g., gas-air interface in bubbles, pond surfaces, proto-cell membranes), wherein low starting concentrations are amplified by progressively lower low water activity. 


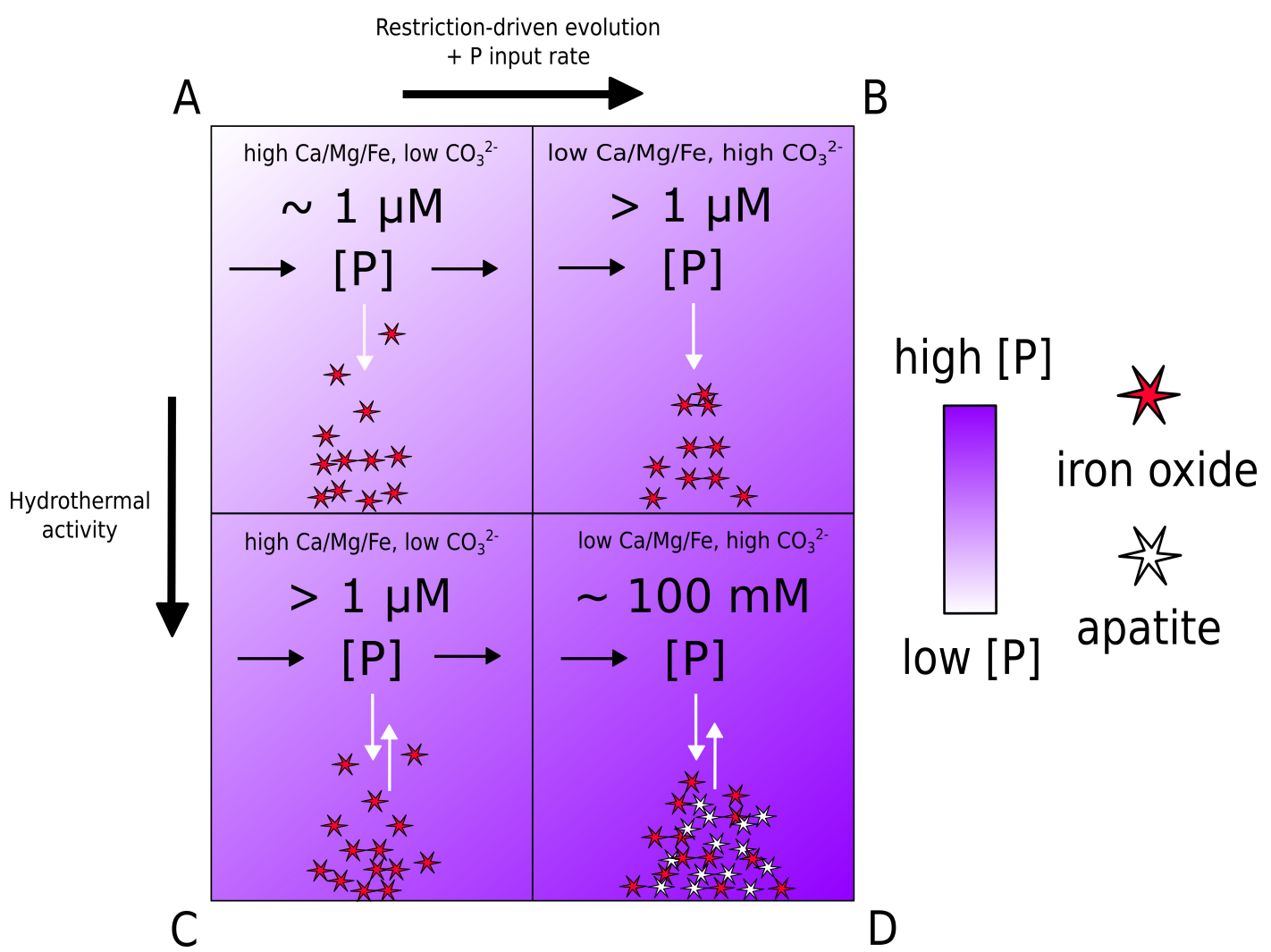

Figure 6. Phosphorus concentrations as a function of environmental variables. Increasing basin restriction and hydrothermal activity will both elevate DIP concentrations for a fixed input and water column sink. Saturation ceilings for phosphate minerals are elevated in restricted basins, allowing significant DIP accumulation if overall sink efficiency is moderated e.g., via hydrothermal activity.

We will now demonstrate how the availability of both DIP and ribose will have exerted a crucial control over the next step in reaction scheme Figure 3: ribose phosphorylation.

\subsection{Ribose phosphorylation}

As explained in section 1.2, we explicitly include $a_{W}$ as a variable in our treatment of dehydration reactions in this work. Thus, the equilibrium constant $(K)$ for ribose phosphorylation via DIP

$$
K 1=\frac{[\text { Ribose phosphate }] a_{W}}{[\text { Ribose }][\mathrm{DIP}]} .
$$

Alternatively, having measured the free energy change of the reaction, we can calculate $K$ as follows

$$
K=e^{\frac{\Delta G}{R T}} .
$$

Where $\Delta \mathrm{G}$ is the free energy change of the reaction $\mathrm{J} \mathrm{mol}^{-1}, \mathrm{R}$ is the universal gas constant $\left(\mathrm{J} \mathrm{mol}^{-1} \mathrm{~K}^{-1}\right)$, and $\mathrm{T}$ is temperature $(\mathrm{K})$.

Our calculations inherently assume that thermodynamic equilibrium is achieved - an assumption unlikely to accurately reflect complex prebiotic environments (i.e., open-system, many sources and sinks, activation energy barriers for reactions). However, whilst data for the overall free energy changes associated with reactions of interest are somewhat widespread, we encountered a dearth of data with which to evaluate reaction kinetics.

Therefore, in order to be consistent in our approach, we calculate reaction equilibria based solely on thermodynamic considerations. This is a situation equivalent to assuming perfect catalysis (e.g., 
Figure 4) and/or considering a closed system following an arbitrarily long approach to equilibrium. Important caveats for this approach are addressed in Section 3.

Equation [5] can be used to calculate $K$ as a function of $T$. However, extrapolation via this equation is only valid if properly calibrated for the free energy of reaction, which may not remain constant across all environmental conditions. In particular, the stability of the products, reactants, and transition state are dependent on the local hydrogen bonding environment, which can vary enormously at low values of $a_{W}$ [46]. Indeed, Nam et al. [23] demonstrated that $\Delta G$ for reaction [4] is negative under the desolvating low $a_{W}$ conditions found at microdroplet air-water interfaces.

Experimental constraints on ribose phosphorylation thermodynamics as a function of $a_{W}$ are limited e.g., [23]. However, the chemical potential of water, which can be related directly to capacity for ion or molecule solvation [47], is logarithmically dependent on water activity, as follows (Guggenheim, 1965):

$$
\mu_{W}=\mu_{W}^{*}+R T \ln a_{W}
$$

Where $\mu_{W}$ is chemical potential of water in solution $\left(\mathrm{J} \mathrm{mol}^{-1}\right)$ and $\mu_{W}^{*}$ is the chemical potential of pure water $\left(\mathrm{J} \mathrm{mol}^{-1}\right)$ at standard state.

Table 1. Thermodynamic data for ribose phosphorylation experiments.

\begin{tabular}{ccc}
$\begin{array}{c}\text { Conditions } \\
\text { Reference }\end{array}$ & $\Delta G(\mathrm{~kJ} / \mathrm{mol})$ & Total ionic strength (M) \\
\hline \hline $\begin{array}{c}\text { Solvated } \\
\text { Camici et al (1980) }\end{array}$ & 5.4 & 0.11 \\
\hline $\begin{array}{c}\text { Desolvated } \\
\text { Nam et al (2017) }\end{array}$ & -1.02 & 0.10 \\
\hline \hline
\end{tabular}

We consider two-end member scenarios in order to conservatively bound the parameter space for dehydration reactions at equilibrium: (1) no relationship and (2) a logarithmic relationship between $a_{W}$ and the thermodynamics of ribose phosphorylation. This latter case appears more reasonable than a linear dependence, as desolvation as a behaviour requires near total local elimination of water.

Table 1 reports published free energies of formation for ribose phosphate and water from ribose and phosphate at solvated as well as desolvated conditions. We calculate water activity in the dilute experiment of [48] by assuming an ideal solution, such that Raoult's law can be applied [49]:

$$
a_{W}=1-X_{S}
$$

where $X_{S}$ is the ionic strength of the solution. Assuming 55.5 moles of water in $1 \mathrm{~L}$ of pure water and given the reported average total solute strength of $0.11 \mathrm{M}$ [48], this yields

$$
a_{W}=(55.5-0.11) / 55.5=0.998
$$

We use literature estimates for $a_{W}$ at air-water interfacial regions (Table 1) to bracket the desolvating conditions experimentally and theoretically investigated in air-interfacing microdroplets by Nam et al. [23].

The air-water interface can be described as a transition across which a smooth change in the chemical potential and activity of water takes place [50]. Relating the desolvating conditions in the experiments of Nam et al. [23] to a single value of $a_{W}$ is hence an unrealistic approximation. However, 
we can use the change in water chemical potential across the air-water transition zone to bracket the possible $a_{W}$ values at which desolvation was achieved in the experiments performed by [23].

In order to obtain reasonable estimates for the relationship between $a_{W}$ and desolvation behavior across air-water interfaces, we use an estimate of $a_{W}$ in the equilibrated near-surface vapor phase. These are the minimum values of $a_{W}$ possible during the transition from the liquid to vapor phase (see Supplementary Information, Section 7.1)

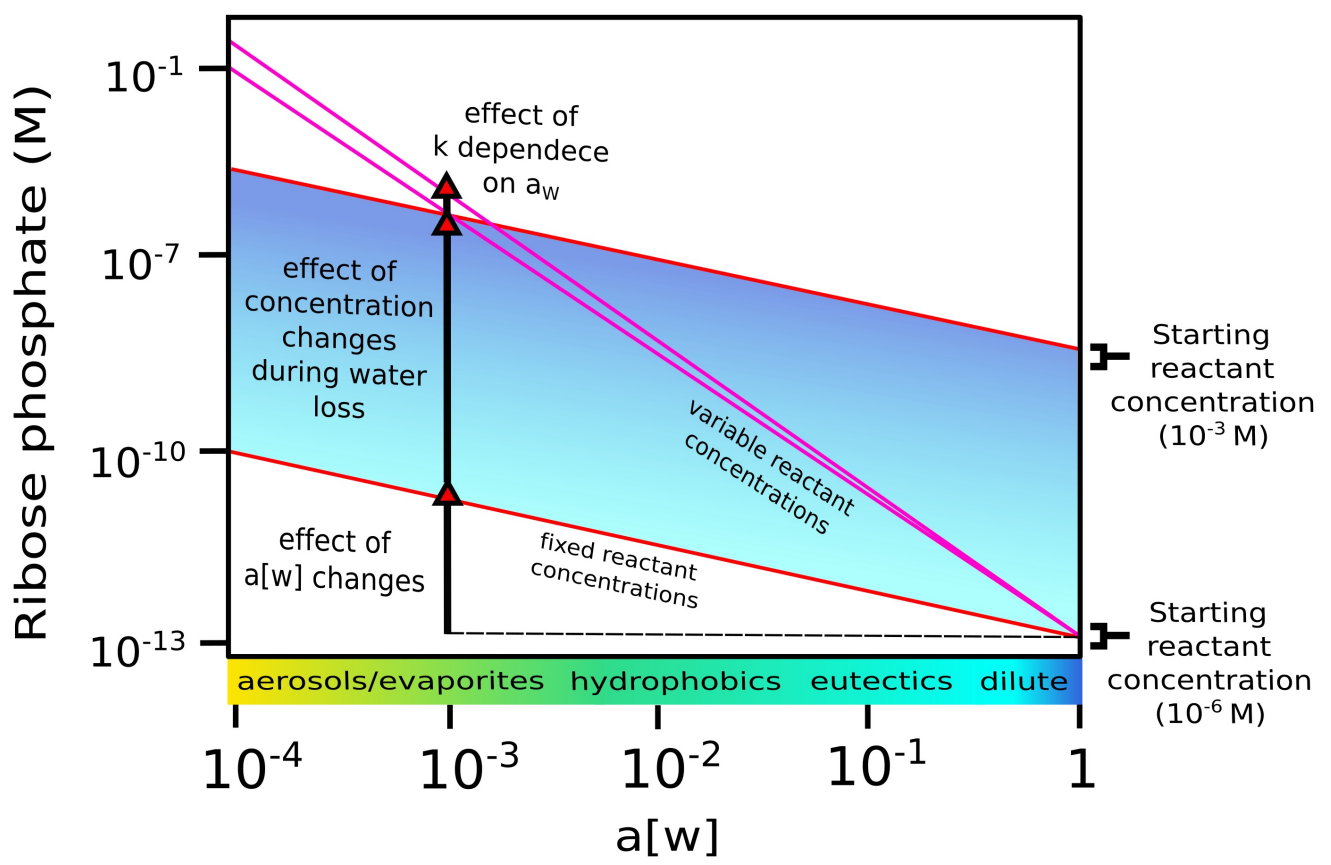

Figure 7. Predicted ribose phosphate yield as a function of water activity, for various equilibrium reactant concentrations and temperatures. Estimated $a_{W}$ parameter space for environments is based on the following references: Damodaran [51], Nam et al. [23]; this work.

Figure 7 plots the equilibrium yield of ribose phosphate as a function of ribose, phosphate, and $a_{W}$. In each case, elevated absolute concentrations of ribose and phosphate are shown to increase the absolute equilibrium concentrations of ribose phosphate. Temperature has a comparatively insignificant effect (not shown). Our parameterization for a dependency of overall reaction energetics on $a_{W}$ produces an effect which becomes more important at only low values of $a_{W}$ ( 1 order of magnitude higher ribose phosphate concentrations at $a_{W}=10^{-4}$ ).

In a prebiotic context, Figure 7 suggests that, for a moderate $\Delta G$ reaction such as [4], low $a_{W}$ environmental conditions may induce ribose phosphate yields of $>50 \%$. However, even the most ideal stabilization pathways for ribose will also yield a number of sugar byproducts (Supplementary Information section 7.2; [27]). These byproducts will then compete with ribose in downstream reaction steps, reducing the equilibrium yield of e.g., desired phosphorylation products. Complex sugar scenarios predict, for any given set of conditions, several orders of magnitude lower ribose-P yields in comparison to ribose-only scenarios (Figure 8). 


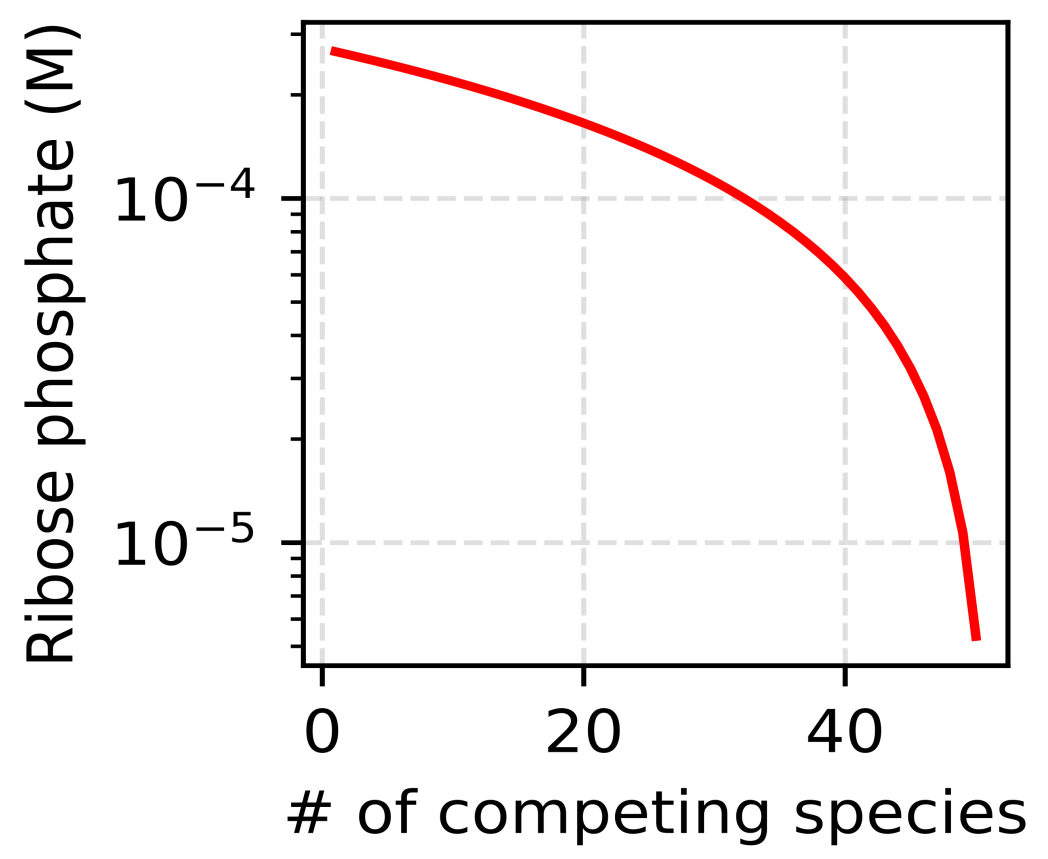

Figure 8. Effect of sugar mixture complexity on ribose phosphate yield during phosphorylation, assuming $1 \mathrm{mM}$ starting concentrations of sugar and phosphate, and assuming equal reaction constants for each sugar species with phosphate (see Supplementary Information section 7.2), with water activity set to $1 \times 10^{-3}$.

Solutions to the issue include sugar-specific stabilization (e.g., via borate; [32]), more efficient ribose synthesis, and/or higher equilibrium concentrations of DIP. Each of these requirements would in turn place specific constraints on the origin scenario via second-order parameter space (see Section $3)$.

More generally, Figure 8 highlights that the successful incorporation of realistic levels of complexity in prebiotic experiments is a key test that proposed origin scenarios must pass.

\subsection{Nucleobase availability}

Nucleobases feature in the third step of our chosen reaction scheme (Figure 3). The nitrogenous chemistry of canonical nucleobase synthesis pathways interfere destructively with the oxygeneous chemistry of the formose reaction. Such problems may well be circumvented by considering alternatives to free nucleobases (e.g., mixed nitrogeneous/oxygeneous chemistry; [2,12,52]), but are beyond the scope of the present article.

Instead, we examine exogenous nucleobase delivery via meteorite deposition. This potential pathway seperates ribose and nucleobase synthesis temporally and spatially, but allows for their later interaction. Pearce et al. [53] estimate that the accumulation of $14 \mu \mathrm{M}$ peak nucleobase concentrations in prebiotic shallow ponds is conceivable via meteoritic delivery followed by evaporative concentration. Concentrations in submarine environments, meanwhile, are negligible.

Reaction of ribose-P and nucleobases has been demonstrated to yield ribonucleotides [24]. However, these experiments were performed using $>>100 \mu \mathrm{M}$ concentrations of both ribose-P and nucleobases. Therefore, several orders of magnitude higher adenine concentrations than those predicted by Pearce et al. [53] are required to connect these two pathways, which may constitute a major problem for the prebiotic plausibility of our selected reaction scheme (Figure 3).

However, constant pore-water outflow (i.e., basal seepage) served to limit nucleobase accumulation during evaporative concentration in the model of Pearce $e t$ al. [53] i.e., the significance of this term increased as nucleobase concentrations increased, thereby offsetting the evaporative 
concentration effect to a degree. In reality, the evaporation of solute-rich solutions will eventually result in mineral precipitation and some fraction of the pond water should become trapped at the surface. Pearce et al. [53] also found that nucleobase photodissociation in the 'dry state' is highly efficient for reasonable early Earth conditions, but did not consider wet state photolysis. However, adenine mixed into the near surface region of a prebiotic pond would likely be exposed to UV irradiation. Indeed, many prebiotic reaction schemes rely on UV driven reaction steps that occur in bulk solution e.g., [7,52,54]. Hence, UV irradiation is an important interference to consider in the plausibility of scenarios in which UV must be present, either as a likely environmental factor and/or as a directly invoked participant in other ongoing chemistry.

We rework the model of Pearce et al. [53] to account for variable outflow and UV shielding in the pond environment, both in the dry and the wet state. This model was designed for adenine specifically, for which Pearce et al. [53] were able to assemble sufficient constraints on meteoritic abundance and degradation rates. Pond dimensions are chosen to reflect those considered in Pearce et al. [53]. Each model scenario starts with a set concentration of total adenine $(10.54 \mu \mathrm{M})$ in a pond of $1 \mathrm{~m}$ radius and depth i.e., corresponding to immediate diffusion of all adenine from a $20 \mathrm{~cm}$ (carbonaceous chondrite type - and hence adenine-rich) impactor. Outflow is then treated as either continuous or halting after $90 \%$ evaporation, with the UV photo-destruction sink activated during the wet or dry stage to model different types of shielding.

It is critical to caveat in our use of this model that selective concentration of adenine is assumed during evaporation, as well as a lack of competing chemical sinks for adenine, and instantaneous transfer of adenine from the impactor to the pond environment. Our model therefore represents an upper estimate on meteoritic adenine concentrations in evaporating ponds. Further details of the reworked model can be found in the Supplementary Information (Section 7.3).

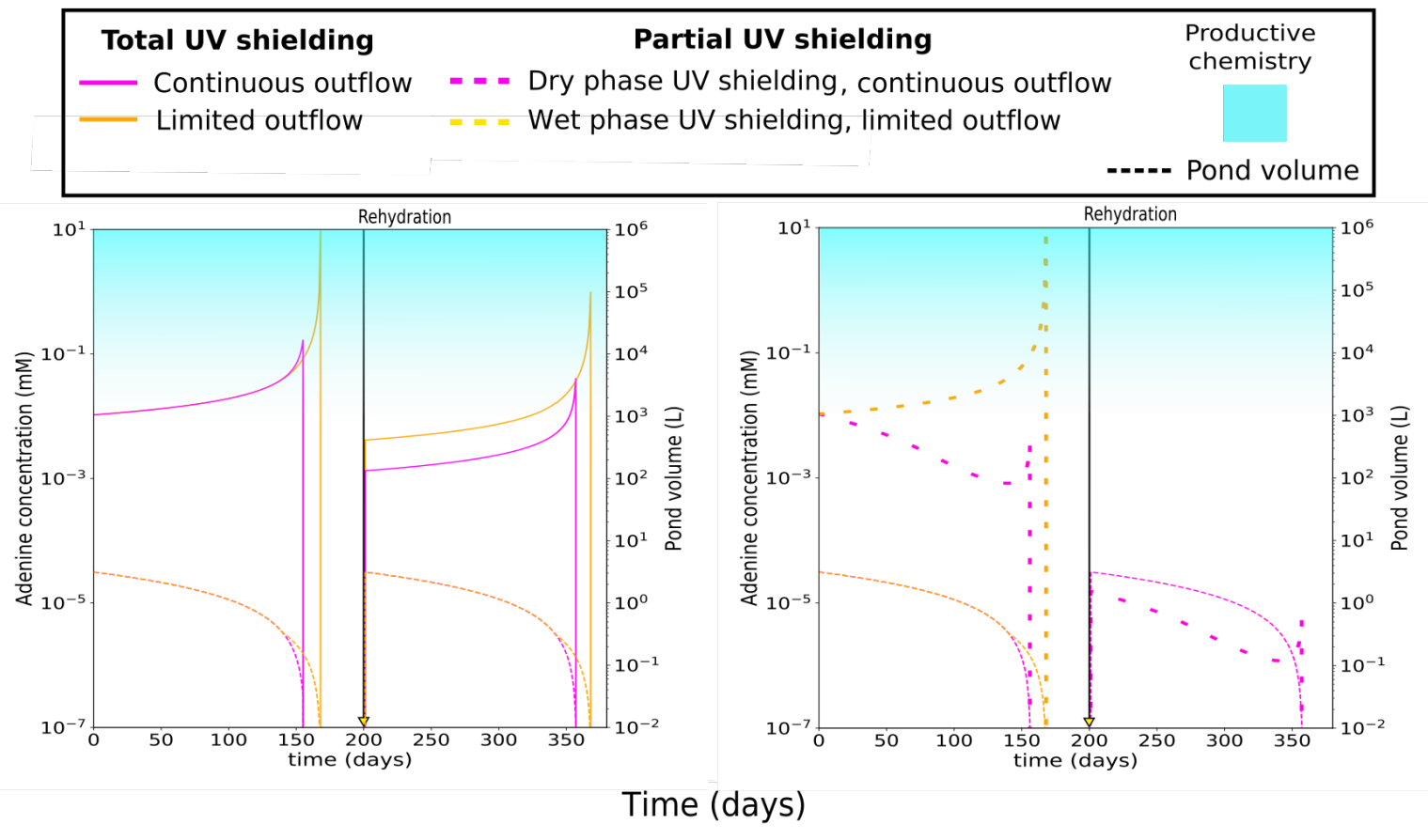

Figure 9. Evaporative concentration of adenine, contrasting conditions of total UV shielding and partial UV shielding. Only scenarios involving limited outflow and total UV shielding during both wet and dry phases are capable of hosting productive prebiotic chemistry following a single deposition of meteoritic nucleobases. In a scenario where UV shielding ceases in the dry stage, nucleobases are totally destroyed and hence are not plotted following the rehydration event at 200 days. 
Figure 9 provides reasonable limits on nucleobase accumulation in prebiotic ponds. Nucleobase concentrations immediately decline from our chosen optimistic starting condition if wet-state photo-destruction is included, prior to a small increase just before full evaporation.

In scenarios where UV shielding is total, many wet/dry cycles take place with non-negligible adenine concentrations. Maximum nucleobase concentrations of $10 \mathrm{mM}$ are achieved given limited outflow, versus a limit of around $1 \mathrm{mM}$ given continuous outflow. Our upper limits on nucleobase accumulation in wet state UV-shielded shallow pond systems overlaps with the concentration ranges ( $>\mathrm{mM}$ ) used by Kim and Benner [24] and Suárez-Marina et al. [25] to promote reaction with ribose phosphate.

However, our results indicate that a given starting complement of nucleobases can only participate in productive prebiotic chemistry over the course of a multiple wet/dry cycles given complete UV shielding. Nucleobases are more rapidly destroyed if UV irradiation is allowed for during the wet stage and are totally destroyed during dry state UV irradiation.

At this stage we do not rule out reaction between ribose-P and nucleobases via a coupled exogeneous-wet/dry cycling pathway. However, we must simultaneously acknowledge the restricted environmental parameter space that such a scenario occupies, requiring rapid deposition/diffusion of adenine from meteoritic material followed by total UV shielding and limited outflow.

\subsection{Ribonucleotide formation}

The glycosylation of ribose phosphate and nucleobases to form ribonucleotides (and other assorted glycosylation products) has been experimentally demonstrated by Suárez-Marina et al. [25]; Figure 3). These reactions were performed using $\mathrm{mM}$ concentrations of sugar phosphate and nucleobases, at low $\mathrm{pH}$, and at elevated temperature, but in the absence of any specific chemical or physical catalysts. glycosylation was driven most efficiently during wet/dry cycles i.e., by low $a_{W}$ conditions. This result is consistent with glycosylation being a dehydration reaction (Figure 3).

Quantitative determinations of the equilibrium constant for this reaction have not yet been made. However, using semi-quantiative yield data published by Suárez-Marina et al. [25], we can determine a semi-quantitative equilibrium constant for use in a reaction network path analysis [7] (Section 3). The equilibrium constant equation for the reaction of adenine and ribose-P to form adenosine monophosphate (AMP) is

$$
K 2=\frac{[\mathrm{AMP}]\left[a_{W}\right]}{[\text { adenine }][\text { ribose phosphate }]} .
$$

We estimate an AMP yield of $<0.1 \%$ at equilibrium in dilute solution. We select a reaction reported by [25] in which $25 \mathrm{mM}$ starting concentrations of adenine and ribose phosphate were used, as follows

$$
\begin{gathered}
a_{W}=\frac{55.5-0.053}{55.5}=0.999 \text {, and } \\
K 2=\frac{[25 * 0.001][0.999]}{[25 * 0.999][25 * 0.999]}=0.04 .
\end{gathered}
$$

Using the above relations, we can derive estimates for AMP equilibrium concentrations as a function of reactant concentrations and $a_{W}$ (Figure 10).

We assume that this semi-quantitative estimate is representative of ribonucleotide formation via glycosylation in general. Without constraints on the sensitivity of glycosylation thermodynamics to desolvation, we cannot apply the same correction obtained for ribose phosphorylation in section 2.3.

It is important to note that our estimate is likely to be an upper limit on AMP yields via this pathway. The experiments of Suárez-Marina et al. [25] produce complex mixtures, rather than pure yields of AMP. Therefore, as with step 3 of Figure 3, a complexity cascade may further impede the efficiency of this step. 
It is clear that AMP formation by the pathway discussed is less favoured than ribose phosphorylation and may therefore constitute a limiting step for eventual RNA formation. Keeping this outcome in mind, we proceed to the final step in our selected RNA synthesis pathway (Figure 3): polymerization of ribonucleotide monomers.

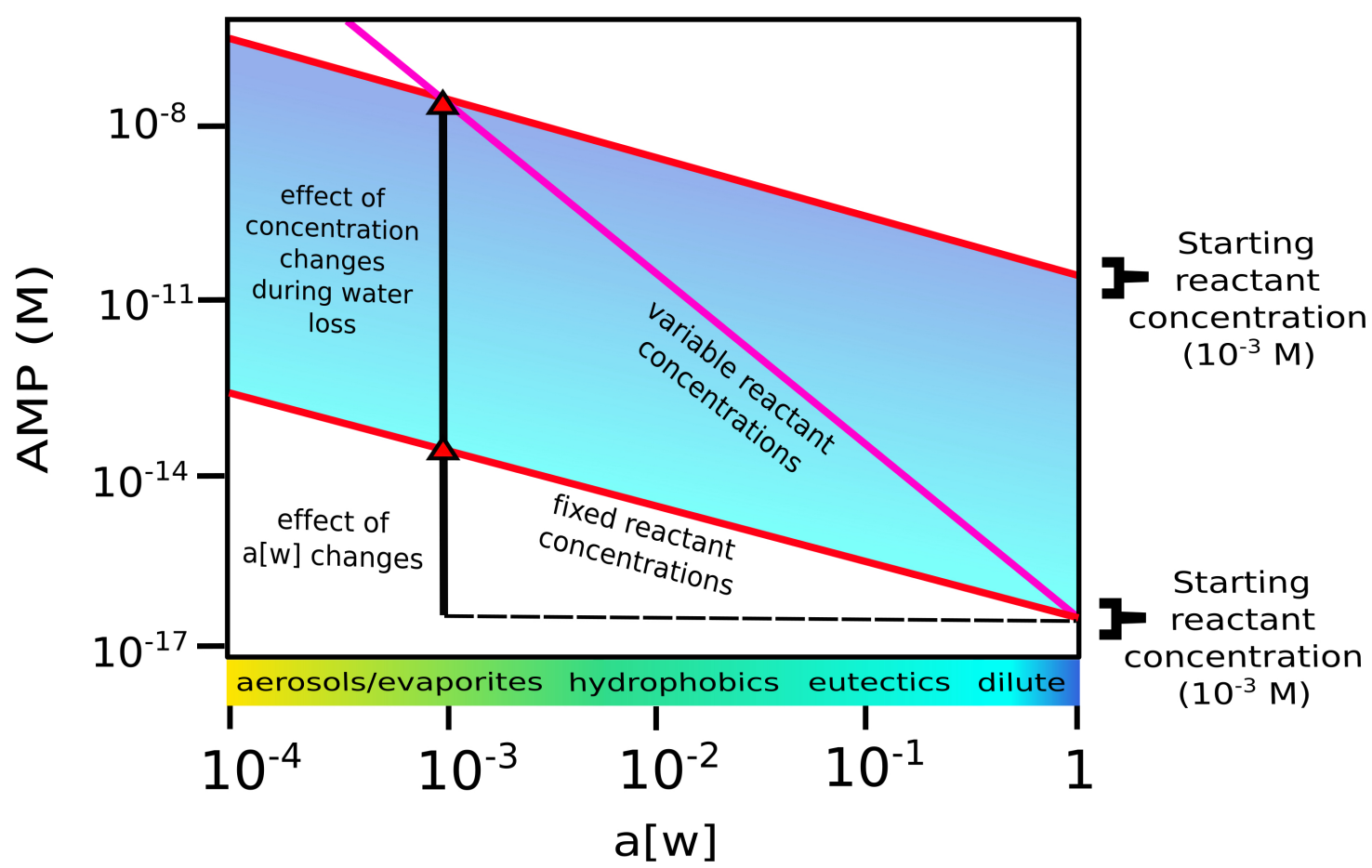

Figure 10. Predicted ribonucleotide (AMP) concentrations as a function of water activity and equilibrium reactant concentrations. All calculations calibrated for $298 \mathrm{~K}$. Estimated $a_{W}$ parameter space for environments is based on the following references: Damodaran [51]; Nam et al. [23], this work.

\subsection{Ribonucleotide polymerization}

We consider the simple case of monomer elaboration via phosphate ribonucleotides (Figure 3). Such reactions have been experimentally and theoretically interrogated under conditions of drying and heating [55]. We extend an isodesmic model for non-cooperative polymerization of RNA (Mast et al. [55] Zhao and Moore [56]) to include the effects of water activity (see Supplementary Information, Section 7.4).

Polymer abundance is calculated as follows

$$
C_{n}=K_{d}^{-1}\left(K_{d}[\mathrm{~A}]\right)^{n},
$$

where $C_{n}$ is concentration (M) of polymer length with $n, K_{d}$ is the monomer-monomer dissociation constant of RNA, and [A] is monomer concentration (M). Given that RNA hydrolyis/polymerisation can be written as a one-step reaction involving the direct addition or removal of water, we assume that the hydrolysis rate of RNA monomer-monomer bonds is directly proportional to $a_{W}$ [57]. Given this assumption, the experimentally determined $K_{d}$ for RNA dissociation by Mast $e t$ al. [55] can be adjusted as follows:

$$
K_{d}^{a_{W}}=\frac{K_{d}}{a_{W}}
$$


Figure 11 plots the relative equilibrium concentrations of polymers of varying length. Even given starting monomer concentrations of $1 \mathrm{M}$, we estimate that 200-mers (the minimum length estimated for self replicating ribozymes; [58]) are only accessible given the near total exclusion of water in this system.

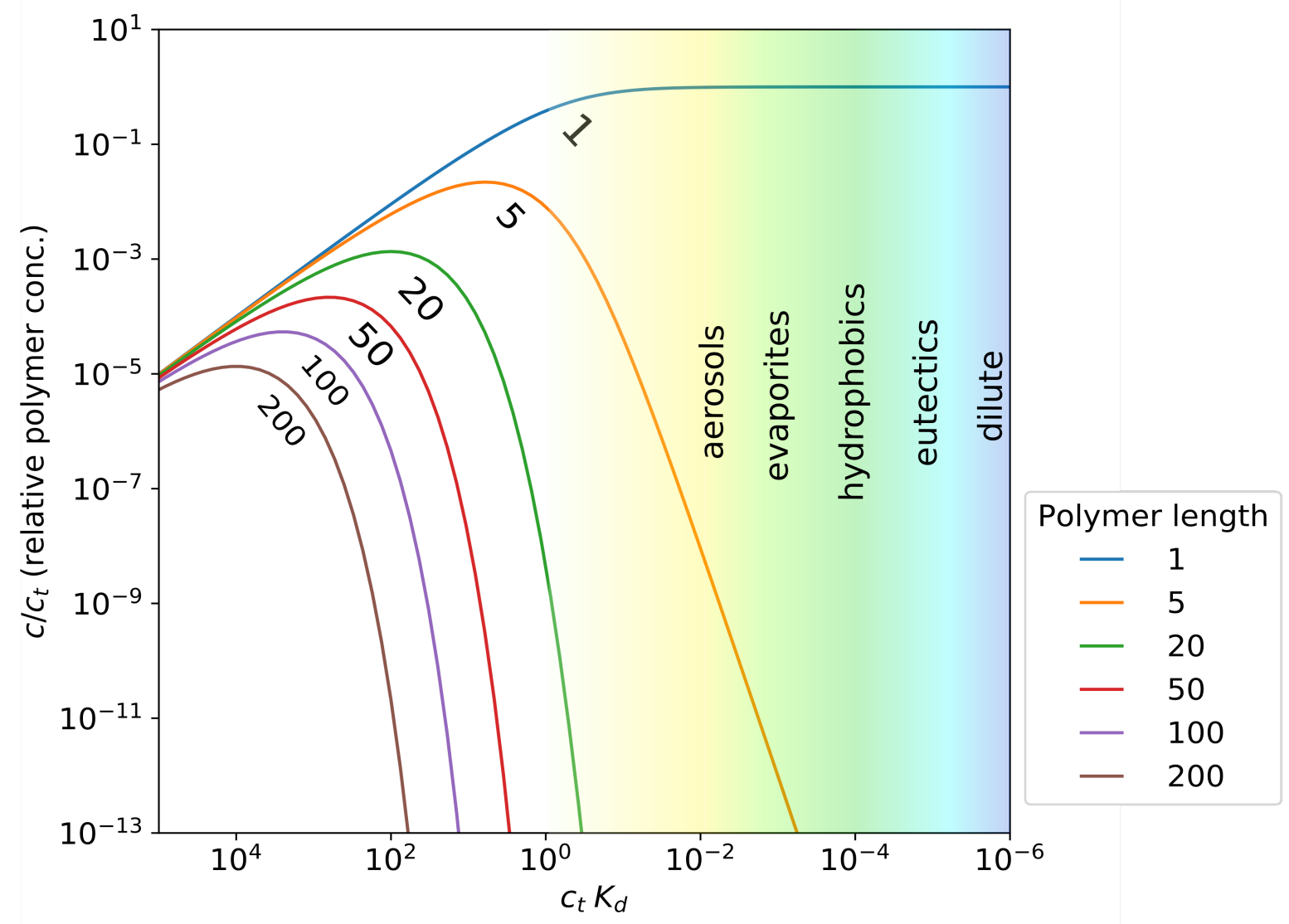

\section{(Polymerisation efficiency)}

Figure 11. Polymer concentrations as a function of polymerisation efficiency i.e., the product of the monomer-monomer dissociation constant, which is set to $1 \times 10^{-5} * a_{W}$, and total monomer concentration. Estimated $a_{W}$ parameter space for environments is based on the following references: Damodaran [51]; Nam et al. [23], this work.

\subsection{Water activity}

We have found that lowered water activity can aid in driving prebiotic chemistry at each stage in the reaction pathway we selected for analysis. However, in order to provide a complete assessment of this pathway, we must consider the environmental dependencies of plausible early Earth settings that have been previously suggested to promote low $a_{W}$ conditions.

In the context of biological water cycling, cells must continuously repair biomolecules (e.g., DNA) against hydrolysis, a behavior that is achieved by cycling between the hydrolyzing and dehydrating microenvironments formed during enzyme-substrate binding [21]. Such continuous cycling results in cell-wide dynamic equilibrium i.e., stabilisation across some timescale of properties, structures, or behaviours that are out of equilibrium with the wider environment e.g., [59]. For example, the $\mathrm{pH}$ gradient that universally characterises cell membranes represents one such dynamic equilibrium. 


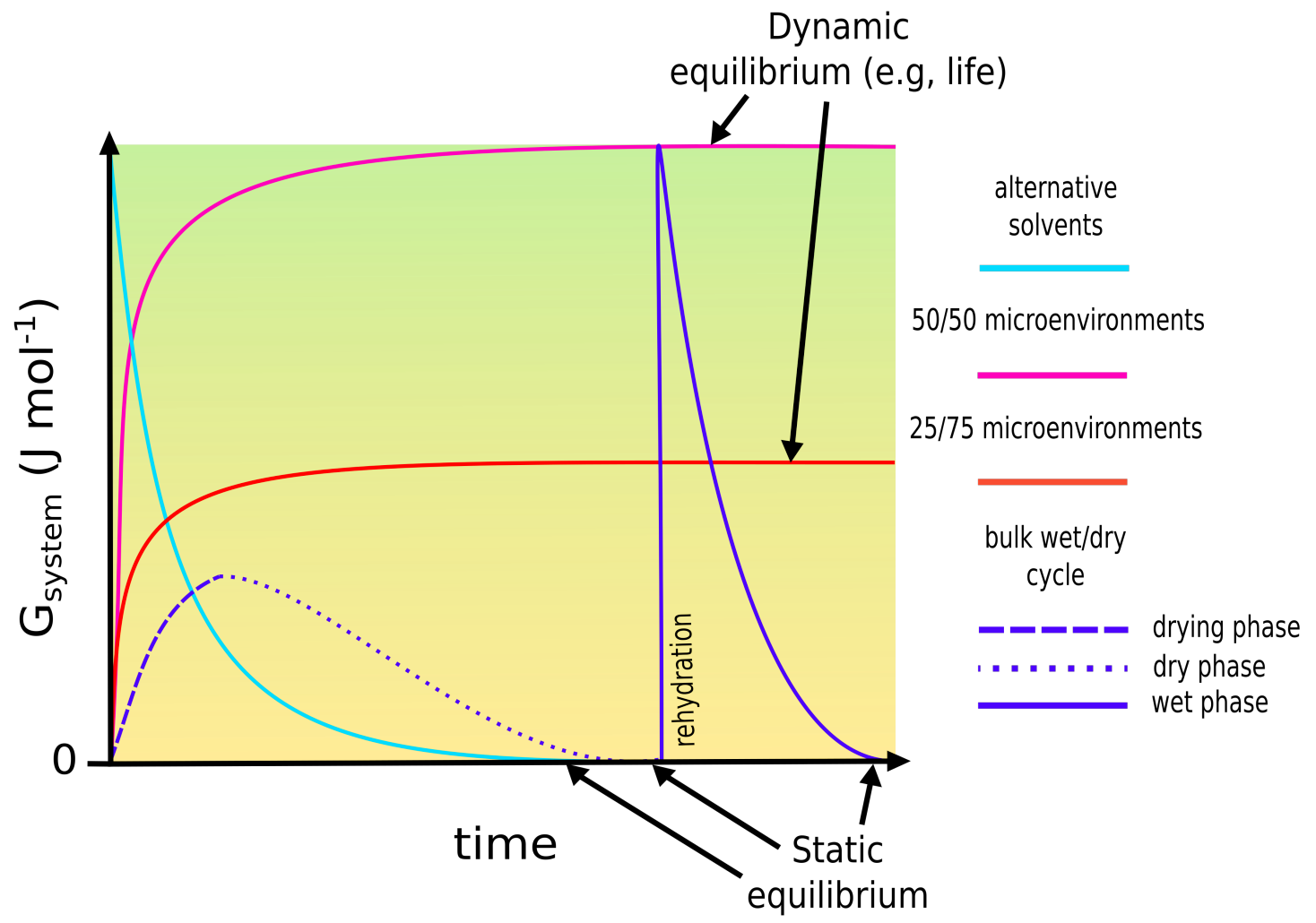

Figure 12. Conceptualisation of trends in free energy in various environments hosting dehydration-hydrolysis reactions. The alternative solvents case assumes input of reactants that then proceed to achieve a static equilibrium. Wet/dry cycles produce a stock-pile of dehydration products in the dry phase, which are then unstable in the wet phase and proceed to static equilibrium. Microenvironments that are in diffusive exchange with their surroundings will set up a dynamic equilibrium, wherein hydrolysis or dehydration products, which are unstable in the bulk versus micro-environment, respectively, are constantly exchanged via diffusion. This is most efficient for heterogeneous 50:50 volume ratios of dehydrating to hydrolysing environments.

Fluxes between hydrolysis and dehydration in the cell are balanced overall, yet free energy is made available to exploit on local scales via microenvironmental shifts [60]. Without the intervention of enzymes, a spatially and temporally homogeneous static equilibrium would be achieved, corresponding to the cessation of metabolism and other key regulatory activities.

Dynamic equilibria are inherently harder to maintain across longer spatial or temporal scales ([61] e.g., limitation of cell volume by the surface area of bioenergetic membranes. The specific time and length-scales imposed on prebiotic dehydration chemistry by individual environments may therefore inhibit some of the steps required in a given reaction scheme, and comprise important interferences to consider when constructing self-consistent models of prebiotic chemistry.

Figure 12 presents a conceptual overview of free energy trends that should be exhibited by the main environments and mechanisms that have been proposed to drive prebiotic dehydration chemistry. Free energy in the system can be treated as the sum of chemical potentials $(\mu)$ and number of moles $(N)$ for all species (i) undergoing reaction, e.g., as in equations 4 and 8:

$$
G_{\text {system }}=\sum_{i} \mu_{\mathrm{i}} N_{i}
$$

In systems that reach static equilibrium, equation 11 will sum to zero. However, other systems may drive balanced yet continuous fluxes of dehydrated and hydrolysed products into hydrolysing 
and dehydrating environments, respectively, resulting in non-zero values of $G_{s y s t e m}$ i.e., a dynamic equilibrium. Conceptually, dynamic equilibrium is most easily ascribed to environments that host active changes of material between water-poor and water-rich micro-environments, as well as life. In contrast, alternative solvents and bulk-wet dry cycles are reliant on larger scale environmental perturbations to maintain their ability to perform chemical work e.g., addition of new chemical building blocks, or the addition or removal of water, respectively.

\section{Evaporites}

Bulk wet/dry cycles, already mentioned in the context of nucleobase concentration (section 2.4), serve to concentrate and desiccate reactants during evaporation. Experiments of this type can rapidly induce complete desiccation via an extended period of heating in the dry phase, and have been shown to promote phosphorylation and polymersation e.g., [62,63].

Wet/dry cycles are conceivable in any subaerial environment (e.g., riverine, hot spring, lake, pond, and so on), although the rate of cycling will inevitably be slower for larger volume environments. An important characteristic of bulk cycling settings is that the free energy pool (product/reactant ratio) is largest immediately following rehydration (Figure 12). Longer-term cycles therefore face the problem of a significant interval for product decomposition prior to any possibility of 'repair' in the dry phase.

\section{Alternative solvents}

Alternative solvents promote dehydration chemistry via their inherently low water activity. Here, chemical constituents of the solvent act as condensing agents which react with water and thus drive forward dehydration reactions e.g., formamide, urea; [16,45]. However, the approach to equilibrium in alternative solvents also requires that free energy in the system will tend to a global minimum over time (Figure 12). At least in principle, this is quite unlike the dynamic disequilibrium maintained by living organisms.

The most tractable implication of this observation is simply that some level of imposed chemical heterogeneity is needed to initiate a dehydration/hydration protometabolism in alternative solvents, which may have been plausibly provided by, for example, interfacing minerals and microenvironments.

A further critique of an alternative solvent scenario is the inherent prebiotic plausibility of obtaining such environments on the early Earth. Widely-used UAFW solutions are only plausible on a macroscopic scale given high atmospheric partial pressures of $\mathrm{HCN}, \mathrm{CO}_{2}$, and $\mathrm{NH}_{3}$ ([64]). These conditions are not presently favoured by state-of-the-art models of early atmospheric chemistry [65]).

The timescale for forming condensing agent eutectic solutions during bulk-scale dry-down is estimated to be $>1000$ years, with only a minimal time available for wet-stage chemistry thereafter i.e., prior to solidification [64]. However, transiently high concentrations of condensing agents have been suggested to occur during wet/dry cycling [64], which may be most readily attained in microscale environments.

\section{Microenvironments}

Prebiotically plausible microenvironments that have so far been demonstrated to promote dehydration chemistry include air-water interfaces [66], mineral surfaces/structures [67], and functionalized protocells ([68]).

Air-water interfaces would have been found at the surface of numerous aqueous prebiotic environments e.g., microdroplets [23], and in gas bubbles [69]. which have been shown to promote peptide and sugar phosphate formation. Similarly, mineral catalysts have been experimentally verified 
to accelerate phosphorylation and polymerization reactions (e.g., schreibersite, clays; $[67,70]$ ), which may relate to the presence of low $a_{W}$ internal or surface cavities $[46,71]$.

Finally, hydrophobic/hydrophilic amphiphiles can generate $a_{W}$ gradients across spontaneously formed microstructures e.g., the bilayers of protocells $[51,72,73]$. To-date, protocell structures have been principally investigated in the context of prebiotic chemistry that would follow in the wake of the RNA world e.g., encapsulation of pre-formed RNA polymers, or the processing of phosphorylated compounds e.g., [74].

However, the prebiotic potential of amphiphiles for other forms of chemistry seems clear: water activities as low as $<0.01$ have been theoretically predicted for the internal hydrophobic spaces of phospholipid bilayers ([51]), and functionalized protocells of this type have been demonstrated to assist in activating other prebiotic components, which may then go on to participate more readily in dehydration reactions [73]. Protocells therefore constitute another valid reaction medium for microscale dehydration reactions.

In many cases, the dehydration products generated in these microscale environments would suffer from diffusive loss when coupled to a wider aqueous environment. Environments which are confined sufficiently to avoid the diffusion problem then face another: access to all of the prebiotic materials needed to explain the origin of life (e.g., products of atmospheric chemistry). This paradox may be solved by considering scenarios where the microenvironment in question constitutes a large fraction of the total volume experiencing diffusion and/or by increasing the cycling rate between hydrolyzing and dehydration environments, analogous to modern biochemistry (Figure 12).

With our exploration of prebiotic parameter space complete, we can now use environmental interferences to evaluate specific origin of scenarios.

\section{Path analysis by interference chemistry}

\subsection{A step-wise assessment}

In the previous section we closely considered the geochemical parameter space of each step in our selected reaction pathway (Figure 3). We will now demonstrate how a deeper consideration of interference chemistry can constrain possible environmental scenarios for this particular reaction path.

At each step, we highlight all of the possible environmental pathways available to host the specified chemistry, assess the significance of environmental interferences in each case, before moving to the next step. Pathways or environments which are ruled out at an earlier stage on the basis of a particular interference do not feature thereafter in our analysis.

\section{Initial conditions}

In Section 2, we concluded that both subaerial and submarine hydrothermal settings could have generated formaldehyde. We limited nucleobase availability to exogeneous delivery, which restricts plausible environments for the origin of life to subaerial restricted basins with total UV shielding (Figure 13, B1), which could be achieved either by a transiently opaque atmosphere, buoyant organic molecules, or ice-cover.

Hydrothermally active end members of restricted systems were also invoked (Figure 13, B1.2-3) in order to maintain Fe-P redox recycling and hence $>100 \mu \mathrm{M}$ DIP concentrations (Figure 6). Neutral-alkaline variants (1.2A-B and 1.3) would stabilise and select for ribose via borate cyclisation (owing to sugar- and phosphate-driven changes in boric acid pKa in the former; Figure 5). However, we must still invoke a driving force behind formose reactions in these environments.

Alkaline basins would support base catalysed formose reactions. Closed basin soda-type hydrothermal systems hosted by ultramafic rocks would support alkaline $\mathrm{pH}$ conditions despite high $\mathrm{pCO}_{2}$ (Figure 13, B1.3). Given low atmospheric $\mathrm{pCO}_{2}$, soda-type alkaline hydrothermal environments could have been hosted in rocks of wider compositional diversity e.g., mafic and intermediate 


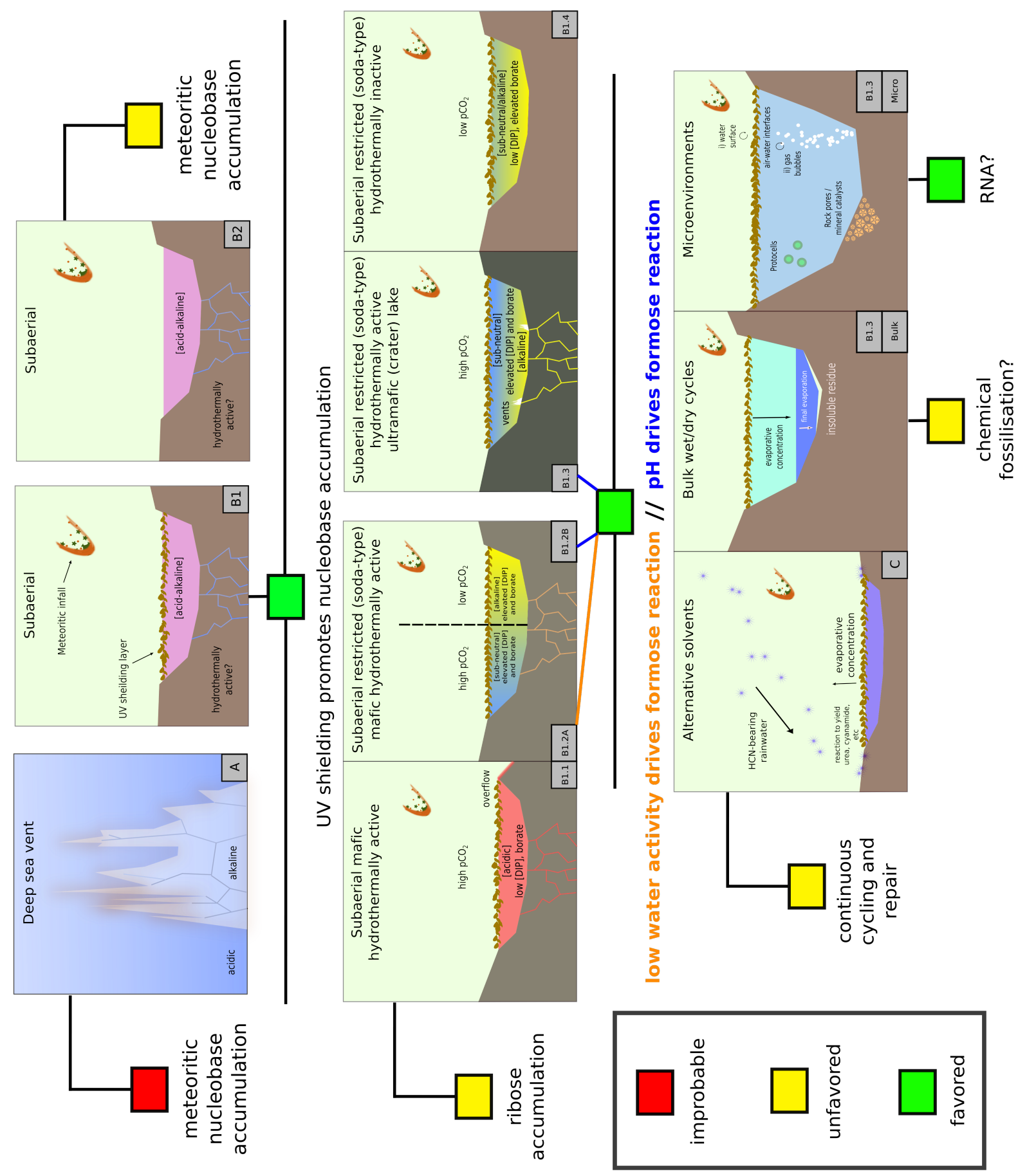

Figure 13. Environmental path analysis for the chemical scenario discussed in the main text. At each stage, arguments (given in the text) are presented that allow us to evaluate which subset of environments are most favored for hosting the chemistry specified in Figure 3. Note: this path analysis is only intended to cover the toy model reaction scheme considered in this manuscript. Specific restrictions include only meteoritic sources of nucleobases, as well as a dependence on direct ribose-phosphate glycosylation 
lithologies (B1.2B). Subsequent synthesis steps to formose reaction and phosphorylation would then rely upon wet/dry cycling of some kind.

We can also consider the possibility that wet/dry cycling itself is sufficient to drive the formose reaction. In this case, any $\mathrm{pH}$ from neutral-to-alkaline is sufficient to enable constructive borate-sugar interferences (1.2A-B, and 1.3).

In all scenarios we consider, wet/dry cycling is inevitably required to drive at least some steps in the synthesis pathway. The key, of course, is doing so selectively. It is here that environmental interference chemistry elucidates critical and novel restrictions in available parameter space.

Figure 13 illustrates the range of environmental variants available to us that could drive, via low $a_{W}$, each of the remaining steps shown in Figure 3. By this point, we have already ruled out many classes of early Earth environments for this particular reaction pathway (Figure 13) and are thus working within a restricted region of geochemical parameter space.

\section{Bulk wet/dry cycles}

One probable geochemical interference in bulk wet/dry cycles is mineral precipitation. Solute-rich aqueous environments will extensively precipitate mineral residues during their evaporation. This process is exemplified by the Si-rich sinter/chert that is widely deposited in hot spring environments [75]. Supersaturation in silica occurs readily in these environments, owing both to high dissolved $\mathrm{Si}$ in the upwelling heated fluid and further concentration during evaporation at the surface [75].

Silica precipitates have been demonstrated to thermally stabilise ribofuranose [76]. Borophosphates, meanwhile, have been shown to specifically aid in the regiospecific phosphorylation of ribonucleosides [32]. In this context, evaporite phases may constitute a constructive interference in prebiotic dehydration chemistry.

However, many such mineral precipitates are extremely insoluble in ambient temperature fluids once desiccated. Indeed, the oldest evidence of life on land is preserved in Si-minerals that precipitated from a hot spring at 3.5 Ga [77]. Given that dissolved silica in particular is thought to have been abundant in the early oceans, rivers, and hydrothermal systems e.g., [77,78], the entrapment of prebiotic materials by Si-precipitates may constitute a widespread and destructive geochemical interference for origin scenarios that rely on bulk wet/dry cycles. On this basis, we show wet/dry cycles as an unfavored pathway for driving dehydration chemistry in Figure 13. This is an important prediction to test experimentally.

\section{Alternative solvents}

Alternative solvents may have accumulated nucleobases, formaldehyde, and phosphate. The inherent low water activity of such environments would have catalyzed the formose reaction to offer high ribose yields. If the solvent composition included a large fraction of condensing agent, then the incorporation of phosphate, reaction with sugars, potential reaction with nucleobases, and polymerization of any ribonucleotides formed, are all potentially facile steps to perform.

However, as acknowledged in Section 2, the reactivity conferred by low $a_{W}$ on formaldehyde is likely to apply also to increasingly complex carbohydrates i.e., the classic tar problem [7]. A similar complexity cascade can be expected for other reaction steps e.g., non-selective production of phosphorylated molecules. As such, potentially crippling levels of self-interference may occur in alternative solvents. We posit this as a challenge to the emergence of life in such environments (Figure 13). 
Microenvironments presents a distinct alternative to both bulk wet/dry and alternative solvent scenarios. The initial conditions we have laid out remain the same: restricted basins with moderate $\mathrm{pH}$ and $\mathrm{T}$ conditions as well as reasonable sources of DIP and formaldehyde. In an ideal scenario of this type, the formose reaction to yield ribose and other sugars is promoted by a high density of low $a_{W}$ microenvironments (Figure 12), which could take any of the forms shown in Figure 13 i.e., protocellular, rock pores/mineral catalysts, and air-water interfaces.

A key advantage of each of these environments is their ability to rapidly and continuously drive wet/dry cycles on a local scale. The transition to low $a_{W}$ permits the efficient reaction of molecules that may only be present at low starting concentrations in the bulk environment. This results from increased activity of solutes in the low $a_{W}$ region. In the case of dehydration reactions, low $a_{W}$ itself also contributes towards driving the reaction forward.

For example, a formose reaction catalyzed by low $a_{W}$ would logically be followed in short order by incipient phosphorylation of the sugar products. Analogous to the borate effect, stable phosphorylated sugars would be produced without the need for destructive high $\mathrm{T}$ or high $\mathrm{pH}$ conditions. However, these two interferences are hardly mutually exclusive. Evaporative wet/dry cycling In Borate-rich environments may precipiate $\mathrm{B}$ - and P-bearing phases e.g., lüneburgite $\left(\mathrm{Mg}_{3} \mathrm{~B}_{2}\left(\mathrm{PO}_{4}\right)_{2}(\mathrm{OH})_{6}-8 \mathrm{H}_{2} \mathrm{O}\right)$. Such phases may be ideal candidates for driving local-scale stabilisation of ribose coupled to targeted phosphorylation. A detailed interrogation of the interference chemistry of mineral interfaces is beyond the scope of this work, but provides a rich topic for future study.

We further predict that cycles of dehydration and hydrolysis chemistry should permit the purification of higher ribose yields than those achieved via dehydration chemistry alone. This argument follows from the relative susceptibility of long-chain sugars to hydrolysis (e.g., [79]). Drying phase chemistry will generate a complex mixture of chained sugars, but the wet phase should react this assemblage back to mainly monomeric species.

We suggest that this cyclic approach will prove advantageous at each stage of the reaction pathway shown in Figure 3. Indeed, the advantages of cyclic dehydration/hydrolysis chemistry has already been been demonstrated for the reaction between ribose phosphate and nucleobases i.e., Figure 3; [25]. A dynamic equilibrium that rapidly cycles back and forth between wet and dry states, guaranteed in high-proportion microenvironment scenarios (12), is a potentially idealized end member of such cyclic purification pathways.

\section{Ideal microenvironment scenarios}

The reaction path shown in Figure 3 is potentially compatible with each of the microscale scenarios overviewed in Figure 13. However, there are again restrictions, inherent to each particular case, that should be carefully considered.

A wide variety of microscale environmental settings have been experimentally investigated in the context of prebiotic chemistry (e.g., mineral pores, ice crystal interfaces, microdroplets, gas bubble interfaces, and various amphiphile-based molecular structures; [23,69,73,74,80,81]).

We reason that the conditions spontaneously generated in these microenvironments can be paralleled (at least crudely) to the low water activities that are critical for enzymatic catalysis of dehydration reactions. In our view, the low $a_{W}$ conditions that have been demonstrated to favour so much constructive prebiotic chemistry may have been generated with a minimal number of associated destructive environmental interferces by microscale environments. However, such scenarios are only competitive if the microscale environment comprises a reasonable fraction of the local environment (i.e., Figure 12), over a sufficient time to allow for complex chemical development.

\subsection{Towards prebiotic reaction networks}

Our path analysis using interference chemistry has so far allowed us to plot a plausible pathway that links each step to the next. From this, several scenarios emerge as reasonable (B1.2A-B, 1.3; Figure 
13). However, these scenarios can only be held up as truly plausible for the reaction pathway we have explored once each step is considered simultaneously i.e., as a chemical network.

The final stage of our analysis takes a first step towards such an integrative reaction network. Our simple treatment is nonetheless rooted in interference chemistry, and allows us to observe important characteristics of origin scenarios at a larger scale. We assume diffusion-limited chemical equilibrium and mass conservation within a model system containing three reaction steps (for which we have earlier established equilibrium constants):

- Ribose phosphate formation

- AMP formation

- RNA polymerisation

We analytically solve the equilibrium constant equations given throughout this work as a simple chemical network (Supplementary Information, section 7.5). Figure 14 presents key results from our approach, showing the equilibrium concentrations of ribose, phosphate, ribose phosphate, adenine, $\mathrm{AMP}$, and RNA polymer concentrations as a function of final water activity in the system. We find that high concentrations of AMP monomers are possible under certain environmentally restrictive conditions, but that achieving high polymerisation efficiency is implausible under current constraints.

It is important to note that water activity in these calculations is inherently limited to $>0.1$, due to the presence of other species that contain $\mathrm{H}_{2} \mathrm{O}$ as a component. Water activity parameter space is thus limited due to the closed system behaviour considered in our analytical solution. Our calculations also assume a system in which water elimination results in concentration of residual chemical components. Owing to this behaviour, we universally observe a steep increase in the concentrations of all other components across a very small range in water activities (less than a single order of magnitude, starting from an initial value of 1). Observed increases are greatest for dehydration products i.e., ribose phosphate and AMP.

We observe differences in the behaviour of the network depending on the equilibrium constants used. When treated as a closed system, the chemical network can only produce RNA monomers as a dominant species when equilibrium constants are used that correspond to reactions taking place in a borate-rich environment coupled to highly efficient glycosylation. This latter environmental-experimental link (B1.X) has yet not been verified for the reaction scheme assessed in this work. This situation represents a narrow region of parameter space e.g., restricted, hydrothermally active, and soda-type basins (14).

Even these idealised results predict that dimers should represent a relatively insignificant fraction of total RNA species. Here, the limitations imposed by closed-system behaviour become important: RNA synthesis is feasible, yet efficient polymerisation is not.

However, open system behaviour is quite conceivable in nature e.g., extended dry phases during wet/dry cycles. Under these conditions, continuous water removal will favour continuous forward reaction in order to attain a new equilibrium. Therefore, open-systems (i.e., those that can experience water loss following a successful dehydration reaction) are able access longer average chain lengths than can be achieved in our closed-system calculations (Figure 15).

The absence of kinetic constraints is another important caveat for the present path analysis. Even the parameter space we have explored that might produce high yields of RNA monomers may be rendered implausible by sluggish forward reaction kinetics given the relatively rapid decay of key reactants. Although not considered here, we note that kinetic considerations may themselves be a powerful corollary to thermodynamic constraints when evaluating scenario plausibility with an interference chemistry approach.

Experimental verification of the relationships between equilibrium constants for dehydration reactions and water activity must also be pursued. For example, we have assumed a linear dependence of RNA monomer-monomer dissociation rate on $a_{W}$, as well as a logarithmic dependence of phosphorylation thermodynamics on $a_{W}$. These are reasonable assumptions given the underlying 


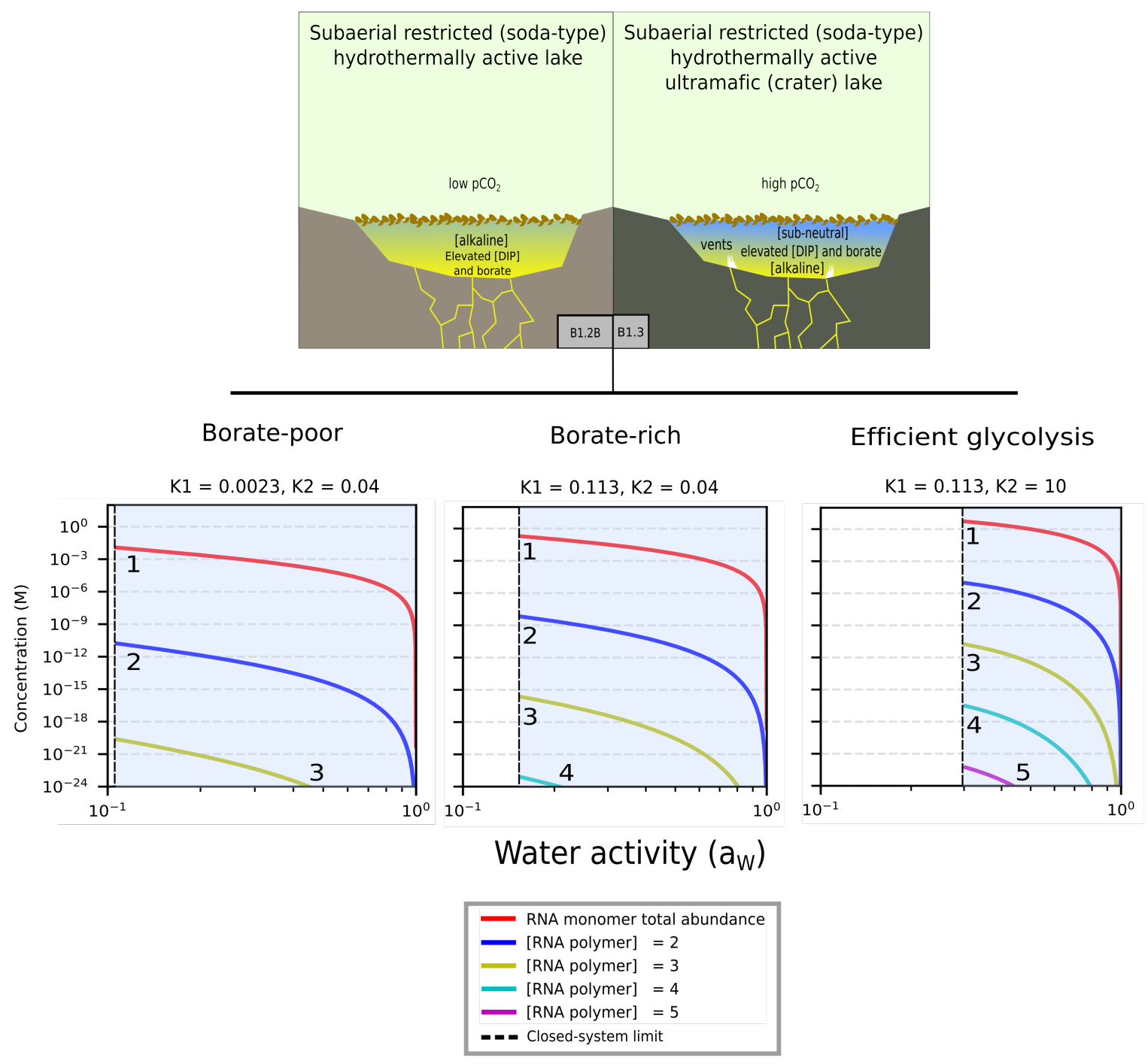

Figure 14. Calculated abundances of RNA polymers from initial AMP monomer concentrations as a function of water activity and solution composition. The initial concentrations of ribose, phosphate, and adenine are equal in all cases. Equilibrium constants are estimated for ribose phosphorylation and glycosylation for three variants of the B1.3 restricted basin scenario, an explanation of which can be found in Figure 13. The third panel refers to a hypothetical, as yet experimentally unverified, scenario in which ribose phosphate glycosylation is highly efficient. Each of these calculations assume a closed system and are thus inherently limited in terms of accessible water activities. 


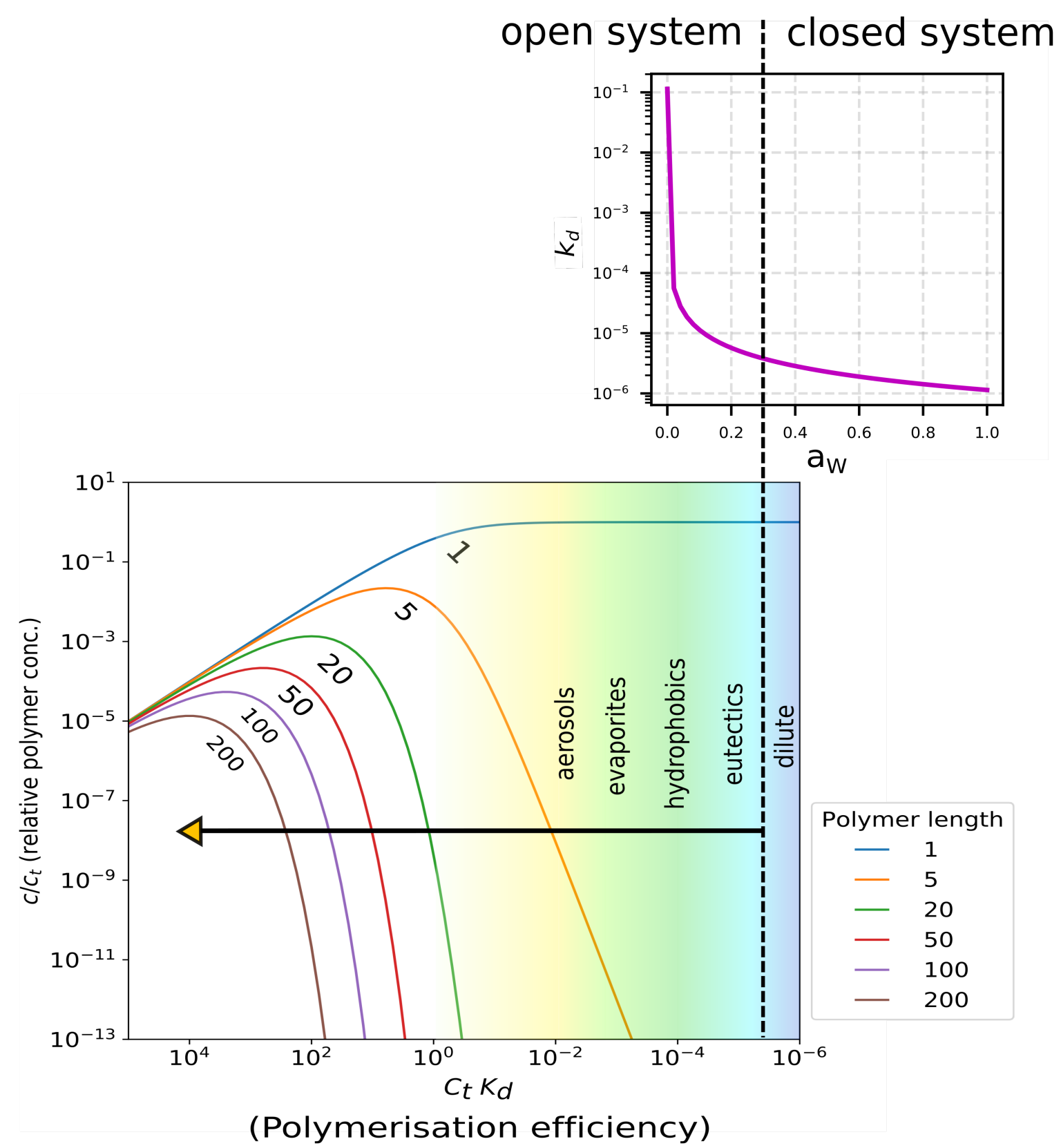

Figure 15. Much of the total parameter space in RNA polymerisation efficiency is only available given open system behavior i.e., where active water loss enables lower equilibrium water activities, which produce higher $K_{d}$ values for RNA monomer-monomer association. Such open system behaviour may occur in micro-environments or during bulk-scale wet dry cycles, for example, as illustrated by the inset scenario diagram - explanations of which can be found in Figure 13. 
physical/chemical mechanisms at play in each case, but strong deviation from such assumed behaviour could lead to different behaviours than we predict.

Despite these caveats, several important and robust outcomes emerge from our work for RNA polymerisation in natural open systems. Firstly, long-term and near-total water exclusion would be needed to achieve and maintain convergence of 200-mer RNA concentrations. Secondly, that highly selective chemistry is needed to enable efficient production and retention of RNA during water-loss.

Hence, the factors that control RNA polymerisation efficiency are vitally important to investigate in more detail. Water activity, solution composition, thermal gradients, and micro-scale structural relationships within the system may all play key roles in governing such dehydration-hydrolysis reactions.

\subsection{Outcomes and caveats}

We find that, via low water activity, conditions might be satisfied that ultimately produce $\mathrm{mM}$ concentrations of RNA monomers. However, with the assumptions in our path analysis, continuous removal of water from the system coupled with highly selective and inherently favoured forward reaction steps is needed to produce convergent concentrations for RNA chain length of even $>2$.

Such idealised reaction conditions may be achieved in a restricted, surficial, phosphate-rich, and alkaline hydrothermal basin (Figure 13), wherein both ribose and AMP may be selectively synthesised and stabilised by readily available borate, which is itself stabilised via constructive sugar interference chemistry. Alkaline conditions may be attained irrespective of host lithology composition at low $p \mathrm{CO}_{2}$, but are reliant on ultramafic hydrothermalism given high $p \mathrm{CO}_{2}$. Wet/dry cycling may then be invoked to cycle to lower water activity and drive further forward reaction. However, any complex mixture will compete with the species considered in this work and thus self-limit the equilibrium yields of dehydration products that may be achieved.

The reliance on meteoritic nucleobases would also seem to be a weak point in the scheme we have analysed (Figure 3). The short time-span over which nucleobases would be available in even the most optimistic exogeneous delivery model places stringent demands on prebiotic chemistry. In our calculations, a single complement of meteoritic nucleobases only experience multiple large-scale wet/dry cycles given total UV shielding (9).

Overall, the reaction scheme we have investigated in this work is highly environmentally sensitive (2) and only appears reasonable under a severely restricted set of conditions. Therefore, we do not claim that the reactions chosen or environmental conditions identified currently constitute a highly plausible pathway to the origin of life. However, our results do demonstrate the ability of interference chemistry to rapidly discriminate between regions of parameter space that constructively and destructively interfere with a particular proposed reaction scheme.

For example, our approach identifies a number of probable destructive (geochemical) interferences that have not previously been widely considered e.g., possible mineral fossilisation during bulk wet/dry cycles. This particular destructive interference is nested within what is otherwise generally considered to be a promising constructive interference in prebiotic reaction schemes: the classic bulk wet/dry cycle. We argue that wet/dry cycling within/across micro-environments, when viewed from an interference chemistry perspective, can be potentially more flexible, selective, and efficient than that which may take place in macro-scale basins.

The specific reaction sequence analyzed here is intended to be representative, rather than final. Our discussion is aimed not at prescribing what was and was not possible in the Prebiotic Era, but provoking future work. Our key result is that interference chemistry provides a useful framework for identifying productive environmental parameter space for prebiotic scenarios.

\section{Summary}

Interference chemistry as parameter space probe 
We investigated the prebiotic plausibility of a single reaction scheme for the production of RNA, under a restricted set of prescribed conditions. In doing so, we identify that environmental interference chemistry can be used to efficiently explore the productive parameter space available to a scenario. Our work both yields specific outcomes of wide applicability to other prebiotic scenarios, as well as highlights issues with the scalability of the approach, which will require a concerted experimental and theoretical effort to address.

We find that low $a_{W}$ should help to drive each of the reactions in Figure 3 i.e., a minimum path from the simplest carbohydrate, through to the RNA world (excluding nucleobase formation). However, low $a_{W}$ may ultimately fail to drive efficient prebiotic chemistry if accessed in the wrong way. That is, destructive interferences resulting from the environmental setting in which low $a_{W}$ is obtained may ultimately preclude the viability of a particular origin scenario e.g., if insufficiently selective, or problematic for the long-term stability/accessibility of products. The specific interferences that we have highlighted are of wide relevance to recent prebiotic reaction schemes e.g., Kim et al. [32], Rimmer et al. [54], Bonfio et al. [68], Morasch et al. [69], Xu et al. [82], Xue et al. [83], Becker et al. [84].

Insights from interference chemistry should prompt reconsideration of which specific environmental parameters may help to drive constructive prebiotic reaction schemes on the early Earth. We have argued that prebiotic experiments are currently designed principally in order to achieve the synthesis of a particular chemical species, or set of species. This approach is often logical, but is inherently biased towards parameter space which affords an ideal or easily observable outcome on the timescale of individual projects.

The interference chemistry concept emphasises the importance of examining the thermodynamic and kinetic fundamentals of each reaction, as well as what aids or impedes these reactions, and indeed sequences of reactions. Doing so helps to more efficiently map out how different reactions interact with one another in nature. This is especially the case on time and spatial scales that are not easily studied in the lab e.g., via extrapolation of integrated prebio-geochemical models.

In order to construct such models, we suggest that a concerted effort should be made to obtain equilibrium, forward, and reverse reaction constants for reactions of interest e.g., Ranjan et al. [85]. For example, sufficiently informative models of sugar cycling of ribose on the early Earth will be impossible to construct without these data.

The demonstrated sensitivity of prebiotic reaction pathways to initial and changing environmental conditions/chemistries further suggests that, on the scale of individual environments, modelling approaches will be much more effective at constraining possibilities than any series of individual experiments. Indeed, as we move towards simulating increasing realistic natural systems, it will become difficult to predict beforehand how the reaction pathway will proceed.

This point seems especially pertinent when we consider that, whilst certain chemistries may appear more beautiful and apparently prebiotic than others, this does not preclude the operation of other chemistry that has been demonstrated as prebiotically plausible. That is, even chemistry which is doomed to produce dead-ends on the road to life may still be of great relevance as a destructive interference, thereby helping to rule out the environments in which it would have occurred.

\section{Prebio-geochemistry: an opportunity for progress?}

In order to tackle the enormous scope of prebiotic chemistry, and in search of a way to organise and utilise metadata in the field, we might turn to the methods employed in deep time biogeochemistry. Here, multi-parameter models are built that attempt to capture the overall behavior of internally complex systems.

Much of the parameter space in such models has yet to be directly verified, for equivalent environments no longer prevail on Earth, or have yet not yet been examined in laboratory and/or (exo)planetary analogue settings [14]. Nonetheless, important aspects of biogeochemical change on 
Earth have been captured in great detail e.g., the rise of surface oxygen levels; [86]. Theoretical models allow such changes to be captured on a range of scales, with predictions made for the most important driving factors.

Model predictions will need to be iteratively tested via laboratory experiments. These may scale from large scale explorations across parameter space [87] to extremely detailed tests of those reaction steps considered to be most critical and/or challenging. Therefore, we highlight some questions and strategies arising from the interference chemistry approach, as follows:

\section{Questions to address}

- How sensitive are prebiotic reaction schemes to initial and evolving conditions?

- Can such dependencies be used to discriminate between specific prebiotic scenarios?

- How do prebiotic systems behave on a variety of time and length scales?

- Which concentrations of reactants, under which conditions, are needed to achieve threshold yields of desired products?

\section{Strategies to consider}

- Perform experiments at varied conditions: test (1) inorganic and organic variables, including $a_{W},(2)$ pressure and temperature conditions - both ambient (at the surface and at depth) and transient (e.g., volcanism, impact events), (3) interferences inherent to an environmental process e.g., mineral 'fossilization' during bulk wet/dry cycles, or UV irradiation of very shallow systems.

- Determine (1) reaction rate and equilibrium reaction constants, (2) reaction free energy changes under various conditions, and (3) the activation energies of forward versus reverse reactions.

- Construction of prebio-geochemical models, which can be used to extrapolate towards conditions, timescales, and system complexities that might not yet have been directly tested in the lab.

We propose that testing of origin scenarios should branch into prebio-geochemistry. This shift will entail asking questions in a different way e.g., [6,7], running experiments that are increasingly reflective of natural geochemical complexity [88], and the design of integrated models for chemical cycling on the early Earth. The data produced will allow the formulation of interference matrices, wherein ideal parameter space for prebiotic reaction schemes can be refined by the successive addition of environmental variables.

Undoubtedly, the prebio-geochemical landscapes that emerge will change rapidly at first. However, unless we begin to translate the myriad scenarios that exist at present into model space, we will find ourselves overwhelmed by chemical possibilities. It is in this format that parameter space will be most fully explored, including more complex multi-environment scenarios and more detailed reaction pathways (e.g., auto-catalysis and -inhibition) than we have considered in this toy model path analysis.

Indeed, it is not only complex geochemistry that may be utilised in an interference chemistry approach. The same concept naturally extends to origins research in the fields of synthetic geobiology and exoplanetary science, which should offer other unique regions of parameter space with which to estimate the plausibility of competing origin scenarios.

Interference chemistry by design should be well equipped to integrate developments in each of these fields into constraints on plausibly prebiotic environmental boundary conditions. Ideally, a modelling approach rooted in fundamental experimental knowledge will be able to rapidly adapt to such changing boundary conditions. It is in this setting that origin scenarios will be directly testable against one another, wherein common models are used to evaluate distinct chemical and environmental pathways. This approach would further give renewed value to the vast amount of work that has been carried out across the past 50 years of origins science, both successful and 'failed', by redefining such work as indicative of either constructive or destructive interference chemistry. 


\section{Acknowledgements}

C. W. acknowledges NERC and UKRI for support through a NERC DTP studentship, grant number NE/L002507/1. P. B. R. is grateful for funding by the Simons Foundation (SCOL Grant \#599634). Thanks also go to all the members of the Universal Life Initiative at the University of Cambridge for helpful discussions during the formative stages of this manuscript. We acknowledge 3 anonymous reviewers for their constructive feedback.

\section{Author contributions}

Conceptualization, Craig Walton, Paul Rimmer and Oliver Shorttle; Data curation, Craig Walton; Formal analysis, Craig Walton, Paul Rimmer and Oliver Shorttle; Funding acquisition, Helen Williams and Oliver Shorttle; Methodology, Craig Walton and Paul Rimmer; Project administration, Paul Rimmer, Helen Williams and Oliver Shorttle; Validation, Craig Walton; Writing - original draft, Craig Walton; Writing - review and editing, Craig Walton, Paul Rimmer, Helen Williams and Oliver Shorttle.

\section{Supplementary Material}

Codes and further data used in this manuscript can be found in the electronic appendix, as well as upon request.

\subsection{Water activity and phosphorylation thermodynamics}

We can estimate the chemical potential and hence water activity of a vapor phase by considering that an equilibrium relationship exists with a dilute liquid water phase. Given this assumption, there will be a point where the chemical potential of the water vapor is equivalent to that of the liquid solution.

This relationship is calculated after [89], where: $\mu_{W(l)}$ and $\mu_{W(v)}$ are the chemical potentials of water $\left(\mathrm{J} \mathrm{mol}^{-1}\right)$ in the liquid and vapor phase, respectively, $\mu^{\circ}{ }_{W(l)}$ and $\mu_{W(v)}^{\circ}$ are the chemical potentials of pure water in a liquid and vapor phase $\left(\mathrm{J} \mathrm{mol}^{-1}\right)$, respectively, and $\frac{P(W)}{P^{\circ}(W)}$ is the ratio of vapor water pressure in bars relative to the pressure of pure water vapor at standard conditions (also equal to vapor water activity $\left.a_{W(v)}\right)$.

$$
\mu_{W(l)}=\mu_{W(v)}=\mu_{W(v)}^{\circ}+R T \ln \left(\frac{P(W)}{P^{\circ}(W)}\right)
$$

In order to solve this equation for $a_{W(v)}$, we first need to calculate $\mu_{W(l)}$ :

$$
\begin{gathered}
a_{W(l)}=\frac{55.5-0.1}{55.5}=0.9982 \\
\mu_{W(l)}=\mu_{W(l)}^{\circ}+R T \ln a_{W}
\end{gathered}
$$

Using [13], where $\mu^{\circ}{ }_{(l)}$ is $-237.14\left(\mathrm{~J} \mathrm{~mol}^{-1}\right), \mathrm{R}$ is $8.314\left(\mathrm{~J} \mathrm{~mol}^{-1} \mathrm{~K}^{-1}\right), \mathrm{T}$ is $298(\mathrm{~K})$, and $\mathrm{P}^{\circ}(\mathrm{W})$ is 1 bar:

$$
\begin{gathered}
\mu_{W(l)}=-237.14+8.3145 * 298 * \ln (55.4)=-241.61\left(\mathrm{~J} \mathrm{~mol}^{-1}\right) \\
\frac{P(W)}{P^{\circ}(W)}=e^{\frac{\mu_{W(l)}-\mu_{W(l)}^{\circ}}{R T}} \\
a_{W(v)}=e^{\frac{-241.61+227.19}{8.314 * 298}}=9.049 * 10^{-10}
\end{gathered}
$$

We have assumed equilibrium in order to apply equation [13], in the process fixing the chemical potential of individual water molecules in the vapor to be equal to the dilute solution. However, 
the activity of water molecules in the vapor phase is calculated to be much lower, which can reasonably explain the apparent desolvation behaviour invoked by [23] to explain enhanced ribose phosphorylation in microdroplets. We hence obtain an estimated logarithmic relationship between $a_{W}$ and ribose phosphorylation thermodynamics $\left(\Delta_{f} G^{\circ}\right)$, using conservative estimates of $a_{W}$ obtained for the desolvating and dilute experiments of [23] and [48], respectively.

$$
\Delta_{f} G^{\circ}=308.34 * \ln \left(a_{w}\right)+5400.6\left(\mathrm{~kJ} \mathrm{~mol}^{-1}\right)
$$

\subsection{Complexity cascades during sugar synthesis}

Our multiple sugar scenario is simplified, considering only the case that multiple sugars competing with ribose via thermodynamically identical phosphorylation reactions. The concentration of each sugar phosphate in the mixture should be identical given this condition:

$$
\text { [Ribose phosphate }]=\left[\mathrm{N}_{\text {th }} \text { sugar phosphate }\right]
$$

The equilibrium constant determined for ribose phosphate alone should also hold for the mixture as follows:

$$
K_{\text {Sugar phosphate }}=\frac{\left(\sum_{i=m}^{n}[\text { Sugar phosphate }]_{\mathrm{m}}+\ldots[\text { Sugar phosphate }]_{\mathrm{n}}\right) a_{W}}{\sum_{i=m}^{n}[\text { Sugar }]_{\mathrm{m}}+\ldots[\text { Sugar }]_{\mathrm{n}}[\mathrm{DIP}]}
$$

And, for a given concentration of total sugar, DIP, and water:

$$
\begin{gathered}
f[\text { Ribose phosphate }]=\frac{[\text { Ribose phosphate }]}{\sum_{i=m}^{n}[\text { Sugar }]_{\mathrm{m}}+\ldots[\text { Sugar }]_{\mathrm{n}}} \\
f[\text { Ribose phosphate }]=\frac{1}{n} \\
{[\text { Ribose phosphate }]=\frac{K_{\text {Sugar phosphate }}\left(\sum_{i=m}^{n}[\text { Sugar }]_{\mathrm{m}}+\ldots[\text { Sugar }]_{\mathrm{n}}\right)[\mathrm{DIP}]}{a_{W} n}}
\end{gathered}
$$

Sugar phosphorylation thermodynamics are of course variable, but (1) not generally beyond \pm 5 $\mathrm{kJ} / \mathrm{mol}$ for monosaccharide and (2) such data are not presently available for many of the abiological sugars produced in the formose reaction [17]. Figure 8 compares the ideal ribose-only scenario (corresponding to perfectly selective formation and/or stabilization pathways) to a progressively more complex reaction mixture. We use $2 \%$ as our lower bound on ribose yield fraction, which corresponds to the yields obtained in the prebiotic synthesis published by [27].

\subsection{Reworked model for evaporative adenine concentration}

\section{Model assumptions}

Our model reworks the calculations of [53]. This previous work presented results mainly for the case of a $1 \mathrm{~m}$ deep and $1 \mathrm{~m}$ radius evaporating pond, which was chosen in order to fit several assumptions: well-mixed adenine in the water column, likelihood of uniform distribution across surface of the Early Earth, and probability of interaction with meteoritic material in strewn fields. In order to fit within the same logical framework, we work with the same pond dimensions. Each calculation assumes a fixed starting concentration of adenine. This value is semi-arbitrary: it is chosen to reflect a maximally optimistic adenine concentration in the initial dilute pond. We consider the case of instantaneous diffusion of adenine following deposition of a carbonaceous meteoroid of $20 \mathrm{~cm}$ radius i.e., the maximum size predicted by [53]. Given this assumption, concentration in the dilute (1 
$\mathrm{m}$ depth) pond is $10.54 \mu \mathrm{M}$. For comparison, adenine reached a maximum concentration of $2 \mathrm{ppm}$ just prior to full evaporation in the model presented by [53].

Hydrolysis is the only nucleobase sink in our model that is directly sensitive to temperature. However, hydrolysis provides only a negligible contribution to the total nucleobase sink. Hence, within the range of plausible surface conditions at which liquid water is stable (i.e., $0-100{ }^{\circ} \mathrm{C}$ ), temperature has a negligible effect on our calculations. We therefore perform our calculations at a reasonable median value: $25^{\circ} \mathrm{C}$.

Evaporation rate is highly temperature dependent. The time available for nucleobase reaction within each wet phase cycle will hence depend on the ambient temperature of the solution. Outflow rate will also control this timescale.

We specifically test how adenine concentrations in the evaporating pond respond to variable seepage and UV penetration. UV irradiation is a key stage of many prebiotic reaction schemes. Whilst UV does not constructively participate in the reaction scheme we have chosen to test, it may still comprise an important destructive interference.

\section{Hydrolysis}

Change in the mass of adenine in the pond due to hydrolysis is calculated using

$$
\frac{d m_{i}}{d t}=-m_{i} y k
$$

where $m_{i}$ is the mass of adenine in the pond $(\mathrm{kg}), y$ is the number of seconds in a day (the length of timestep used in each calculation, and $\mathrm{k}$ is the rate constant for adenine hydrolysis $\left(\mathrm{s}^{-1}\right)$ :

$$
k=10^{\left(\frac{-5902}{T}\right)+8.15} .
$$

Seepage

Basal outflow rate $(\mathrm{O})$ (i.e., via seepage) was used as a constant in [53]:

$$
O=0.0026\left(\mathrm{~m} \mathrm{~d}^{-1}\right) .
$$

We take the same approach, but note that in reality this value is likely sensitive to local lithology and water table.

The nucleobase loss rate via outflow is assumed to scale proportionally to the concentration of nucleobase in the pond at any given time

$$
\frac{d m_{i(o u t)}}{d t}=O A C_{i}
$$

Where $A$ is pond surface area $\left(\mathrm{m}^{2}\right)$ and $\left(C_{i}\right)$ is nucleobase concentration $\left(\mathrm{kg} \mathrm{m}^{-3}\right)$ at a given time.

\section{Photodestruction}

Dry state:

Photodestruction rates of adenine in the dry state are determined using

$$
\frac{d m_{i(p-d)}}{d t}=M_{i} A
$$

Where $M_{i}$ is the photodestruction flux constant of adenine $\left(\mathrm{kg} \mathrm{m}^{-2} \mathrm{~d}^{-1}\right)$.

The photodestruction flux constant is calculated using 


$$
M_{i}=\frac{\phi F \lambda y \mu_{i}}{h c \mathrm{~N}_{\mathrm{A}}}
$$

where $\phi$ is the quantum efficiency of photodecomposition of the molecules (molecules per photon), $\mathrm{F}$ is the UV flux incident on the pond $\left(\mathrm{W} \mathrm{m}^{-2} \mathrm{~s}^{-1}\right), \lambda$ is the average wavelength of $\mathrm{UV}$ radiation incident on the sample $(\mathrm{m})$, for which we use a value of $(225 \mathrm{~nm}$, the average of the range considered by Poch et al. [90]), $\mu_{i}$ is the molecular weight of the irradiated molecules $\left(\mathrm{kg} \mathrm{mol}^{-1}\right), h$ is Planck's constant $\left(\mathrm{kg} \mathrm{m}^{-2} \mathrm{~s}^{-1}\right), \mathrm{c}$ is the speed of light $\left(\mathrm{m} \mathrm{s}^{-1}\right)$, and $\mathrm{N}_{\mathrm{A}}$ is Avogadro's number (molecules per mole).

\section{Wet state:}

Photodestruction rates of adenine in the wet state is determined following the methods of Zafiriou and True (1979a, 1979b), as well as more recently [85]. We assume that adenine is lost at a rate equal to half the surface photolysis rate down to its photic depth and that the loss rate is 0 below this threshold. We assume that the photic zone for adenine is not well mixed with underlying water during the course of a day, but that concentrations are fully homogenised in the pond by the next day. This approach avoids the need for a continuous diffusion model, but will slightly underestimate the loss rate of adenine as a result.

We also assume that the atmosphere is transparent to UV i.e., the adenine photolysis rate constant determined for irradiation in space at 1 AU by [91] is reasonable. In reality, atmospheric attenuation of short wavelengths will occur and the rate constant we use must thus represent an upper limit on adenine photo-destruction. Our analysis is clearly incomplete. However, the underestimation of loss rates due to ignoring diffusion and the overestimation resulting from using a space-calibrated photolysis rate constant should cancel out (at least to an extent). Our estimate therefore does provides a useful guide for, if not a fully quantitative description of, adenine lifetimes in evaporating ponds. Our approach also serves to highlight critical details that more detailed future models should seek to address.

We calculate the wet-state photolysis rate of adenine via

$$
\frac{d m_{i(p-w)}}{d t}=m_{i} \frac{\Psi}{d} \frac{K_{u v}}{2} .
$$

Where $K_{u v}$ is the photodestruction constant of adenine $\left(\right.$ day $\left.^{-1}\right)$. We use a value of $6.57 * 10^{-2}$, from [91]. Our estimate for the photic depth of adenine ( $\Psi$, units of $\mathrm{m}$ ) is semi-arbitrary. We are guided by the deep penetration of UV-A,B light into organic carbon poor freshwater systems $(>10 \mathrm{~m}$ for $<1$ $\mathrm{mg} \mathrm{L}^{-1}$ dissolved organic carbon) [92]. Assuming an organic rich solution chemistry (i.e., in which prebiotic chemistry might occur), attenuation of UV-C (which should be more easily attenuated) is likely to occur in less than $1 \mathrm{~m}$.

We therefore select $0.5 \mathrm{~m}$ as our photic depth of adenine. We assume a constant transmission profile of UV light throughout the wet/dry cycle, although increasing concentrations of dissolved organic species in the pond will likely result in more realistic time-dependent profiles. This is hence another important and clearly flawed assumption that should be subject to sensitivity tests in future models that focus on the interplay between UV transmission, prebiotic chemistry, and physical attributes of restricted basin environments on the early Earth.

Where photic depth exceeds the depth of the pond, a revised expression is used

$$
\frac{d m_{i(p-w)}}{d t}=m_{i} \frac{K_{u v}}{2} .
$$

We model a scenario involving wet state shielding by only switching on photodestruction after complete evaporation of the pond water. Conversely, UV shielding in the dry state only is modelled by switching off photodestruction after complete evaporation. Rehydration is set to occur every 200 days. 


\subsection{Theoretical basis of RNA polymerisation model}

We extend an isodesmic model for non-cooperative polymerization of RNA [56] [55] to include the effects of water activity. In an isodesmic model, monomer addition equilibrium constants $(k)$ are all identical, such that, where $A_{i}$ is the concentration of a species $i$, and $C_{t}$ is the total concentration of all monomers in the system

$$
\begin{gathered}
{\left[\mathrm{A}_{\mathrm{i}}\right]=k^{-1}(k[\mathrm{~A}])^{i}} \\
C_{t}=\sum_{i+j=n}^{\infty} i k^{-1}(k[\mathrm{~A}])^{i}=\frac{[\mathrm{A}]}{(1-k[\mathrm{~A}])^{2}}(k[\mathrm{~A}]<1) \\
\mathrm{k}[\mathrm{A}]=1+\frac{1}{2 k C_{t}}-\left(\frac{1}{k C_{t}}+\frac{1}{4\left(k C_{t}\right)^{2}}\right)^{1 / 2} .
\end{gathered}
$$

Thus, we can calculate monomer concentration [A], where $K_{d}$ is the RNA dissociation constant determined by [55], [dimer] $/[\text { monomer] }]^{2}$, units $\mathrm{M}^{-1}, n$ is polymer length, and $C_{t}$ is the total monomer concentration $=C_{1}+2 * C_{2}+3 * C_{3} \ldots$, as follows

$$
[\mathrm{A}]=\frac{1}{K_{d}}\left(1+\frac{1}{2\left(K_{d} C_{t}\right)}-\left(\frac{1}{\left(K_{d} C_{t}\right)}+\frac{1}{4\left(K_{d} C_{t}\right)^{2}}\right)^{1 / 2}\right) .
$$

We calculate polymer abundance $\left(C_{n}\right)$ as follows

$$
C_{n}=K_{d}^{-1}\left(K_{d}[\mathrm{~A}]\right)^{n} .
$$

\subsection{Analytical solution for chemical network at equilibrium}

We set up and analytically solve a series of equations for our simple reaction network. The formation of ribose phosphate and AMP are directly connected, whereas AMP polymerisation is post-processed i.e., the calculated equilibrium concentration of AMP is used as the input value of $\mathrm{Ct}$ for polymer speciation calculations. In the following equations, $\mathrm{P}_{\mathrm{i}}$ refers to inorganic dissolved phosphate, $R$ is ribose, $P_{i} R$ is ribose phosphate, $H_{2} O$ is water, $A$ is adenine, and $P_{i} R A$ is AMP, and [X] is the normalized concentration of species $\mathrm{X}$, in units of $\mathrm{M} / \mathrm{M}$.

The reactions we consider, with equilibrium constants, are:

$$
\begin{array}{rr}
\mathrm{P}_{\mathrm{i}}+\mathrm{R} \rightleftharpoons \mathrm{P}_{\mathrm{i}} \mathrm{R}+\mathrm{H}_{2} \mathrm{O}, & K_{1}=\frac{\left[\mathrm{P}_{\mathrm{i}} \mathrm{R}\right]\left[\mathrm{H}_{2} \mathrm{O}\right]}{\left[\mathrm{P}_{\mathrm{i}}\right][\mathrm{R}]} ; \\
\mathrm{P}_{\mathrm{i}} \mathrm{R}+\mathrm{A} \rightleftharpoons \mathrm{P}_{\mathrm{i}} \mathrm{RA}+\mathrm{H}_{2}, & K_{2}=\frac{\left[\mathrm{P}_{\mathrm{i}} \mathrm{RA}\right]\left[\mathrm{H}_{2} \mathrm{O}\right]}{\left[\mathrm{P}_{\mathrm{i}} \mathrm{R}\right][\mathrm{A}]} ;
\end{array}
$$

with the initial $(t=0)$ conditions for $\mathrm{P}_{\mathrm{i}}, \mathrm{R}$ and $\mathrm{A}$ of $\left[\mathrm{P}_{\mathrm{i}}\right]_{0},[\mathrm{R}]_{0}$ and $[\mathrm{A}]_{0}$, respectively:

$$
\left[\mathrm{P}_{\mathrm{i}}\right]_{0}=[\mathrm{R}]_{0}=[\mathrm{A}]_{0} .
$$

All other $t=0$ conditions are set to zero. This establishes the following constraints throughout $t \geq 0$, and therefore when equilibirium is achieved, of:

$$
\begin{aligned}
{\left[\mathrm{P}_{\mathrm{i}}\right]_{0} } & =\left[\mathrm{P}_{\mathrm{i}}\right]+\left[\mathrm{P}_{\mathrm{i}} \mathrm{R}\right]+\left[\mathrm{P}_{\mathrm{i}} \mathrm{RA}\right], \\
{[\mathrm{R}]_{0} } & =[\mathrm{R}]+\left[\mathrm{P}_{\mathrm{i}} \mathrm{R}\right]+\left[\mathrm{P}_{\mathrm{i}} \mathrm{RA}\right], \\
{[\mathrm{A}]_{0} } & =[\mathrm{A}]+\left[\mathrm{P}_{\mathrm{i}} \mathrm{RA}\right] .
\end{aligned}
$$


We also impose local mass balance (i.e. no evaporation), so:

$$
1=\left[\mathrm{P}_{\mathrm{i}}\right]+\left[\mathrm{P}_{\mathrm{i}} \mathrm{R}\right]+\left[\mathrm{P}_{\mathrm{i}} \mathrm{RA}\right]+[\mathrm{A}]+\left[\mathrm{H}_{2} \mathrm{O}\right] .
$$

Because of the initial constraints of Eq. (36) and the stochiometry of Eq. (34):

$$
\left[\mathrm{P}_{\mathrm{i}}\right]=[\mathrm{R}] .
$$

Hence, we can write two equations purely in terms of $\left[\mathrm{P}_{\mathrm{i}}\right],\left[\mathrm{P}_{\mathrm{i}}\right]_{0}$, and $\left[\mathrm{H}_{2} \mathrm{O}\right]$ :

$$
\begin{aligned}
{\left[\mathrm{H}_{2} \mathrm{O}\right] } & =\frac{1}{2}\left(1-2\left[\mathrm{P}_{\mathrm{i}}\right]-\left[\mathrm{P}_{\mathrm{i}}\right]_{0}+\sqrt{\left(2\left[\mathrm{P}_{\mathrm{i}}\right]+\left[\mathrm{P}_{\mathrm{i}}\right]_{0}-1\right)^{2}-4 K_{1}\left[\mathrm{P}_{\mathrm{i}}\right]^{2}}\right), \\
{\left[\mathrm{P}_{\mathrm{i}}\right]_{0} } & =\left[\mathrm{P}_{\mathrm{i}}\right]+\frac{K_{1}\left[\mathrm{P}_{\mathrm{i}}\right]^{2}}{\left[\mathrm{H}_{2} \mathrm{O}\right]}+\frac{K_{1} K_{2}\left[\mathrm{P}_{\mathrm{i}}\right]^{3}}{\left[\mathrm{H}_{2} \mathrm{O}\right]^{2}}+\frac{K_{1}^{2} K_{2}\left[\mathrm{P}_{\mathrm{i}}\right]^{4}}{\left[\mathrm{H}_{2} \mathrm{O}\right]^{3}} .
\end{aligned}
$$

We can solve these equations and use these solutions to solve for remaining unknowns as per earlier expressions:

$$
\begin{aligned}
{\left[\mathrm{P}_{\mathrm{i}} \mathrm{R}\right] } & =\frac{K_{1}\left[\mathrm{P}_{\mathrm{i}}\right]^{2}}{\left[\mathrm{H}_{2} \mathrm{O}\right]}, \\
{\left[\mathrm{P}_{\mathrm{i}} \mathrm{RA}\right] } & =\frac{K_{2}\left[\mathrm{P}_{\mathrm{i}}\right]_{0}\left[\mathrm{P}_{\mathrm{i}} \mathrm{R}\right]}{K_{2}\left[\mathrm{P}_{\mathrm{i}} \mathrm{R}\right]+\left[\mathrm{H}_{2} \mathrm{O}\right]^{\prime}} \\
{[\mathrm{A}] } & =[\mathrm{A}]_{0}-\left[\mathrm{P}_{\mathrm{i}} \mathrm{RA}\right], \\
{\left[\mathrm{P}_{\mathrm{i}}\right] } & =\left[\mathrm{P}_{\mathrm{i}}\right]_{0}-\left[\mathrm{P}_{\mathrm{i}} \mathrm{R}\right]-\left[\mathrm{P}_{\mathrm{i}} \mathrm{RA}\right] .
\end{aligned}
$$




\section{References}

1. Yadav, M.; Kumar, R.; Krishnamurthy, R. Chemistry of Abiotic Nucleotide Synthesis. Chemical Reviews 2020, 120, 4766-4805. doi:10.1021/acs.chemrev.9b00546.

2. Sutherland, J.D. The Origin of Life-Out of the Blue. Angewandte Chemie International Edition 2015, 55, 104-121. doi:10.1002/anie.201506585.

3. Eschenmoser, A. The search for the chemistry of life's origin. Tetrahedron 2007, 63, 12821-12844. doi:10.1016/j.tet.2007.10.012.

4. E., O.L. Prebiotic Chemistry and the Origin of the RNA World. Critical Reviews in Biochemistry and Molecular Biology 2004, 39, 99-123. doi:10.1080/10409230490460765.

5. Haldane, J. The Origins of Prebiological Systems and of their Molecular Matrices. Part I: PERSPECTIVES I 1965, pp. 11-18. doi:10.1016/b978-1-4832-2861-7.50011-9.

6. Wu, L.F.; Sutherland, J.D. Provisioning the origin and early evolution of life. Emerging Topics in Life Sciences 2019, 3, ETLS20190011. doi:10.1042/etls20190011.

7. Benner, S.A.; Bell, E.A.; Biondi, E.; Brasser, R.; Carell, T.; Kim, H.; Mojzsis, S.J.; Omran, A.; Pasek, M.A.; Trail, D. When Did Life Likely Emerge on Earth in an RNA-First Process? ChemSystemsChem 2020, 2. doi:10.1002/syst.201900035.

8. Oparin, A.I. CHEMISTRY AND THE ORIGIN OF LIFE. R.I.C Reviews 1969, pp. 1-12.

9. Orgel, L.E. Is Cyanoacetylene Prebiotic? Origins of life and evolution of the biosphere 2002, 32, $279-281$. doi:10.1023/a:1016509009433.

10. Benner, S.A. Prebiotic plausibility and networks of paradox-resolving independent models. Nature Communications 2018, 9, 5173. doi:10.1038/s41467-018-07274-y.

11. Ruiz-Mirazo, K.; Briones, C.; Escosura, A.d.l. Prebiotic Systems Chemistry: New Perspectives for the Origins of Life. Chemical Reviews 2014, 114, 285-366. doi:10.1021/cr2004844.

12. Islam, S.; Powner, M.W. Prebiotic Systems Chemistry: Complexity Overcoming Clutter. Chem 2017, 2, 470-501. doi:10.1016/j.chempr.2017.03.001.

13. Pasek, M.A.; Gull, M.; Herschy, B. Phosphorylation on the early earth. Chemical Geology 2017. doi:10.1016/j.chemgeo.2017.11.008.

14. Sasselov, D.D.; Grotzinger, J.P.; Sutherland, J.D. The origin of life as a planetary phenomenon. Science Advances 2020, 6, eaax3419. doi:10.1126/sciadv.aax3419.

15. Campbell, T.D.; Febrian, R.; McCarthy, J.T.; Kleinschmidt, H.E.; Forsythe, J.G.; Bracher, P.J. Prebiotic condensation through wet-dry cycling regulated by deliquescence. Nature Communications 2019, 10, 4508. doi:10.1038/s41467-019-11834-1.

16. Hulshof, J.; Ponnamperuma, C. Prebiotic Condensation Reactions in an aqueous medium: a review of condensing agents. Origins of life 1976, 7, 197-224.

17. Pasek, M.A. Thermodynamics of Prebiotic Phosphorylation. Chemical Reviews 2019. doi:10.1021/acs.chemrev.9b00492.

18. Ostwald, W.Z. . Phys. Chem. 1894, 15, 705-706.

19. Wolfenden, R.; Snider, M.J. The Depth of Chemical Time and the Power of Enzymes as Catalysts. Accounts of Chemical Research 2001, 34, 938-945. doi:10.1021/ar000058i.

20. Liu, Z.; Rossi, J.C.; Pascal, R. How Prebiotic Chemistry and Early Life Chose Phosphate. Life 2019, 9, 26. doi:10.3390/life9010026.

21. Benkovic, S.J.; Hammes-Schiffer, S. A Perspective on Enzyme Catalysis. Science 2003, 301, 1196-1202. doi:10.1126/science.1085515.

22. Alberty, R.A. A short history of the thermodynamics of enzyme-catalyzed reactions. J Biol Chem 2004, 27, -. doi:doi:10.1074/jbc.X400003200.

23. Nam, I.; Lee, J.K.; Nam, H.G.; Zare, R.N. Abiotic production of sugar phosphates and uridine ribonucleoside in aqueous microdroplets. Proceedings of the National Academy of Sciences 2017, 114, 12396-12400. doi:10.1073/pnas.1714896114. 
24. Kim, H.J.; Benner, S.A. Prebiotic stereoselective synthesis of purine and noncanonical pyrimidine nucleotide from nucleobases and phosphorylated carbohydrates. Proceedings of the National Academy of Sciences 2017, 114, 11315-11320. doi:10.1073/pnas.1710778114.

25. Suárez-Marina, I.; Abul-Haija, Y.M.; Turk-MacLeod, R.; Gromski, P.S.; Cooper, G.J.T.; Olivé, A.O.; Colón-Santos, S.; Cronin, L. Integrated synthesis of nucleotide and nucleosides influenced by amino acids. Communications Chemistry 2019, 2, 28. doi:10.1038/s42004-019-0130-7.

26. Shapiro, R. Prebiotic ribose synthesis: A critical analysis. Origins Life Evol Biosphere 1988, 18, 71-85. doi:https://doi.org/10.1007/BF01808782.

27. Kim, H.J.; Ricardo, A.; Illangkoon, H.I.; Kim, M.J.; Carrigan, M.A.; Frye, F.; Benner, S.A. Synthesis of Carbohydrates in Mineral-Guided Prebiotic Cycles. Journal of the American Chemical Society 2011, 133, 9457-9468. doi:10.1021/ja201769f.

28. Powner, M.W.; Gerland, B.; Sutherland, J.D. Synthesis of activated pyrimidine ribonucleotides in prebiotically plausible conditions. Nature 2009, 459, 239-242. doi:10.1038/nature08013.

29. Cleaves, H.J. The prebiotic geochemistry of formaldehyde. Precambrian Research 2008, 164, 111-118. doi:10.1016/j.precamres.2008.04.002.

30. Larralde, R.; Robertson, M.P.; Miller, S.L. Rates of decomposition of ribose and other sugars: implications for chemical evolution. Proceedings of the National Academy of Sciences 1995, 92, 8158-8160. doi:10.1073/pnas.92.18.8158.

31. Ricardo, A.; Carrigan, M.A.; Olcott, A.N.; Benner, S.A. Borate Minerals Stabilize Ribose. Science 2004, 303, 196-196. doi:10.1126/science.1092464.

32. Kim, H.; Furukawa, Y.; Kakegawa, T.; Bita, A.; Scorei, R.; Benner, S.A. Evaporite Borate-Containing Mineral Ensembles Make Phosphate Available and Regiospecifically Phosphorylate Ribonucleosides: Borate as a Multifaceted Problem Solver in Prebiotic Chemistry. Angewandte Chemie International Edition 2016, 55, 15816-15820. doi:10.1002/anie.201608001.

33. Toner, J.D.; Catling, D.C. A carbonate-rich lake solution to the phosphate problem of the origin of life. Proceedings of the National Academy of Sciences 2020, 117, 883-888. doi:10.1073/pnas.1916109117.

34. Schubert, D., Boron Oxides, Boric Acid, and Borates. In Kirk-Othmer Encyclopedia of Chemical Technology; American Cancer Society, 2011; pp. 1-68, [https: / / onlinelibrary.wiley.com/doi/pdf/10.1002/0471238961.0215181519130920.a01.pub3]. doi:10.1002/0471238961.0215181519130920.a01.pub3.

35. Morse, J.W.; Mackenzie, F.T. . Aquatic Geochemistry, 4, $301-319$. doi:https://doi.org/10.1023/A:1009632230875.

36. Hao, J.; Sverjensky, D.A.; Hazen, R.M. A model for late Archean chemical weathering and world average river water. Earth and Planetary Science Letters 2017, 457. doi:10.1016/j.epsl.2016.10.021.

37. Kadoya, S.; Krissansen-Totton, J.; Catling, D.C. Probable Cold and Alkaline Surface Environment of the Hadean Earth Caused by Impact Ejecta Weathering. Geochemistry, Geophysics, Geosystems 2020, 21. doi:10.1029/2019gc008734.

38. Yan, J.; Springsteen, G.; Deeter, S.; Wang, B. The relationship among pKa, pH, and binding constants in the interactions between boronic acids and diols-it is not as simple as it appears. Tetrahedron 2004, 60, 11205-11209. doi:10.1016/j.tet.2004.08.051.

39. Catanzaro, E.J. Boric acid: isotopic and assay standard reference materials. ational Bureau of Standards, Institute for Materials Research. 1970, 260.

40. Kim, J.D.; Yee, N.; Nanda, V.; Falkowski, P.G. Anoxic photochemical oxidation of siderite generates molecular hydrogen and iron oxides. Proceedings of the National Academy of Sciences 2013, 110, 10073-10077. doi:10.1073/pnas.1308958110.

41. Herschy, B.; Chang, S.J.; Blake, R.; Lepland, A.; Abbott-Lyon, H.; Sampson, J.; Atlas, Z.; Kee, T.P.; Pasek, M.A. Archean phosphorus liberation induced by iron redox geochemistry. Nature Communications 2018, 9, 1346. doi:10.1038/s41467-018-03835-3.

42. Pasek, M.A. Phosphorus volatility in the early Solar nebula. Icarus 2019, 317, 59-65. doi:10.1016/j.icarus.2018.07.011.

43. Johnson, B.R.; Tostevin, R.; Gopon, P.; Wells, J.; Robinson, S.A.; Tosca, N.J. Phosphorus burial in ferruginous $\mathrm{SiO} 2-$ rich Mesoproterozoic sediments. Geology 2019. doi:10.1130/g46824.1. 
44. Hardie, L.A.; Eugster, H.P. THE EVOLUTION OF CLOSED-BASIN BRINES. Min. Soc. America Spec. Pap. 1970, 3, 273-290.

45. Burcar, B.; Pasek, M.; Gull, M.; Cafferty, B.J.; Velasco, F.; Hud, N.V.; Menor-Salván, C. Darwin\&apos;s Warm Little Pond: A One-Pot Reaction for Prebiotic Phosphorylation and the Mobilization of Phosphate from Minerals in a Urea-Based Solvent. Angewandte Chemie International Edition 2016, 55, 13249-13253. doi:10.1002/anie.201606239.

46. Grommet, A.B.; Feller, M.; Klajn, R. Chemical reactivity under nanoconfinement. Nature Nanotechnology 2020, 15, 256-271. doi:10.1038/s41565-020-0652-2.

47. Lynden-Bell, R.M.; Giovambattista, N.; Debenedetti, P.G.; Head-Gordon, T.; Rossky, P.J. Hydrogen bond strength and network structure effects on hydration of non-polar molecules. Physical Chemistry Chemical Physics 2010, 13, 2748-2757. doi:10.1039/c0cp01701a.

48. Camici, M.; Sgarrella, F.; Ipata, P.L.; Mura, U. The standard gibbs free energy change of hydrolysis of $\alpha$-d-ribose 1-phosphate. Archives of Biochemistry and Biophysics 1980, 205, 191-197. doi:10.1016/0003-9861(80)90098-3.

49. Miyawaki, O.; Saito, A.; Matsuo, T.; Nakamura, K. Activity and Activity Coefficient of Water in Aqueous Solutions and Their Relationships with Solution Structure Parameters. Bioscience, Biotechnology and Biochemistry 2014, 61, 466-469. doi:10.1271/bbb.61.466.

50. Pascal, T.A.; Goddard, W.A. Interfacial Thermodynamics of Water and Six Other Liquid Solvents. The Journal of Physical Chemistry B 2014, 118, 5943-5956. doi:10.1021/jp410861h.

51. Damodaran, S. Water activity at interfaces and its role in regulation of interfacial enzymes: a hypothesis. Colloids and Surfaces B: Biointerfaces 1998, 11, 231-237. doi:10.1016/s0927-7765(98)00040-x.

52. Ritson, D.; Sutherland, J.D. Prebiotic synthesis of simple sugars by photoredox systems chemistry. Nature Chemistry 2012, 4, 895. doi:10.1038/nchem.1467.

53. Pearce, B.K.D.; Pudritz, R.E.; Semenov, D.A.; Henning, T.K. Origin of the RNA World: The Fate of Nucleobases in Warm Little Ponds. PNAS 2017, [1710.00434]. doi:10.1073/pnas.1710339114.

54. Rimmer, P.B.; Xu, J.; Thompson, S.J.; Gillen, E.; Sutherland, J.D.; Queloz, D. The origin of RNA precursors on exoplanets. Science Advances 2018, 4, eaar3302. doi:10.1126/sciadv.aar3302.

55. Mast, C.B.; Schink, S.; Gerland, U.; Braun, D. Escalation of polymerization in a thermal gradient. Proceedings of the National Academy of Sciences 2013, 110, 8030-8035. doi:10.1073/pnas.1303222110.

56. Zhao, D.; Moore, J.S. Nucleation-elongation: a mechanism for cooperative supramolecular polymerization. Organic \& Biomolecular Chemistry 2003, 1, 3471-3491. doi:10.1039/b308788c.

57. Izgu, E.C.; Fahrenbach, A.C.; Zhang, N.; Li, L.; Zhang, W.; Larsen, A.T.; Blain, J.C.; Szostak, J.W. Uncovering the Thermodynamics of Monomer Binding for RNA Replication. Journal of the American Chemical Society 2015, 137, 6373-6382. doi:10.1021/jacs.5b02707.

58. Robertson, M.P.; Joyce, G.F. The Origins of the RNA World. Cold Spring Harbor Perspectives in Biology 2012, 4, a003608. doi:10.1101/cshperspect.a003608.

59. Boekhoven, J.; Hendriksen, W.E.; Koper, G.J.M.; Eelkema, R.; Esch, J.H.v. Transient assembly of active materials fueled by a chemical reaction. Science 2015, 349, 1075-1079. doi:10.1126/science.aac6103.

60. Hanson, R.W. The Role of ATP in Metabolism. Biochemical Education 1989, 2, 86-92.

61. Wrzesinski, K.; Rogowska-Wrzesinska, A.; Kanlaya, R.; Borkowski, K.; Schwämmle, V.; Dai, J.; Joensen, K.E.; Wojdyla, K.; Carvalho, V.B.; Fey, S.J. The Cultural Divide: Exponential Growth in Classical 2D and Metabolic Equilibrium in 3D Environments. PLOS ONE 2014, 9, e106973. doi:10.1371/journal.pone.0106973.

62. CHENG, C.; FAN, C.; WAN, R.; TONG, C.; MIAO, Z.; CHEN, J.; ZHAO, Y. PHOSPHORYLATION OF ADENOSINE WITH TRIMETAPHOSPHATE UNDER SIMULATED PREBIOTIC CONDITIONS. Origins of Life and Evolution of the Biosphere, 32, 219-224. doi:https:/ / doi.org/10.1023/A:1016513114799.

63. Mamajanov, I.; MacDonald, P.J.; Ying, J.; Duncanson, D.M.; Dowdy, G.R.; Walker, C.A.; Engelhart, A.E.; Fernández, F.M.; Grover, M.A.; Hud, N.V.; Schork, F.J. Ester Formation and Hydrolysis during Wet-Dry Cycles: Generation of Far-from-Equilibrium Polymers in a Model Prebiotic Reaction. Macromolecules 2014, 47, 1334-1343. doi:10.1021/ma402256d.

64. Lago, J.L.; Burcar, B.T.; Hud, N.V.; Febrian, R.; Mehta, C.; Bracher, P.J.; Atlas, Z.D.; Pasek, M.A. The Prebiotic Provenance of Semi-Aqueous Solvents. Origins of Life and Evolution of Biospheres 2020, pp. 1-14. doi:10.1007/s11084-020-09595-9. 
65. Rimmer, P.B.; Rugheimer, S. Hydrogen Cyanide in Nitrogen-Rich Atmospheres of Rocky Exoplanets. Icarus 2019, 329, 124-131, [1902.08022]. doi:10.1016/j.icarus.2019.02.020.

66. Griffith, E.; Vaida, V. In situ observation of peptide bond formation at the water-air interface. PNAS, 109, 15697-15701. doi:doi/10.1073/pnas.1210029109.

67. Georgelin, T.; Jaber, M.; Onfroy, T.; Hargrove, A.A.; Costa-Torro, F.; Lambert, J.F. Inorganic Phosphate and Nucleotides on Silica Surface: Condensation, Dismutation, and Phosphorylation. The Journal of Physical Chemistry C 2013, 117, 12579-12590. doi:10.1021/jp402437p.

68. Bonfio, C.; Russell, D.A.; Green, N.J.; Mariani, A.; Sutherland, J.D. Activation chemistry drives the emergence of functionalised protocells. Chemical Science 2020. doi:10.1039/d0sc04506c.

69. Morasch, M.; Liu, J.; Dirscher, C.F.; Ianeselli, A.; Kühnlein, A.; Le Vay, K.; Schwintek, P.; Islam, S.; Corpinot, M.K.; Scheu, B.; Dingwell, D.B.; Schwille, P.; Mutschler, H.; Powner, M.W.; Mast, C.B.; .; Braun, D. Heated gas bubbles enrich, crystallize, dry, phosphorylate and encapsulate prebiotic molecules. Nature Chemistry 2019, pp. -. doi:doi.org/10.1038/s41557-019-0299-5.

70. Pasek, M.; Herschy, B.; Kee, T.P. Phosphorus: a Case for Mineral-Organic Reactions in Prebiotic Chemistry. Origins of Life and Evolution of Biospheres 2015, 45, 207-218. doi:10.1007/s11084-015-9420-y.

71. Swadling, J.B.; Coveney, P.V.; Greenwell, H.C. Clay Minerals Mediate Folding and Regioselective Interactions of RNA: A Large-Scale Atomistic Simulation Study. Journal of the American Chemical Society 2010, 132, 13750-13764. doi:10.1021/ja104106y.

72. Morris, C.E. How did cells get their size? The Anatomical Record 2002, 268, 239-251. doi:10.1002/ar.10158.

73. Deamer, D. Liquid crystalline nanostructures : organizing matrices for non-enzymatic nucleic acid polymerization. Chemical Society Reviews 2012, 41, 5375-5379. doi:10.1039/c2cs35042d.

74. Bonfio, C.; Godino, E.; Corsini, M.; Biani, F.F.d.; Guella, G.; Mansy, S.S. Prebiotic iron-sulfur peptide catalysts generate a $\mathrm{pH}$ gradient across model membranes of late protocells. Nature Catalysis 2018, 1, 616-623. doi:10.1038/s41929-018-0116-3.

75. White, D.E.; Brannock, W.; Murata, K. Silica in hot-spring waters. Geochimica et Cosmochimica Acta 1956, 10, 27-59. doi:10.1016/0016-7037(56)90010-2.

76. Georgelin, T.; Jaber, M.; Fournier, F.; Laurent, G.; Costa-Torro, F.; Maurel, M.C.; Lambert, J.F. Stabilization of ribofuranose by a mineral surface. Carbohydrate Research 2015, 402. doi:10.1016/j.carres.2014.07.018.

77. Djokic, T.; Kranendonk, M.J.V.; Campbell, K.A.; Walter, M.R.; Ward, C.R. Earliest signs of life on land preserved in ca. 3.5 Ga hot spring deposits. Nature Communications 2017, 8, ncomms15263. doi:10.1038/ncomms15263.

78. Lalonde, S.V.; Amskold, L.; McDermott, T.R.; Inskeep, W.P.; Konhauser, K.O. Chemical reactivity of microbe and mineral surfaces in hydrous ferric oxide depositing hydrothermal springs. Geobiology 2007, 5, 219-234. doi:10.1111/j.1472-4669.2007.00113.x.

79. Ebikade, E.; Lym, J.; Wittreich, G.; Saha, B.; Vlachos, D.G. Kinetic Studies of Acid Hydrolysis of Food Waste-Derived Saccharides. Industrial E Engineering Chemistry Research 2018, 57, 17365-17374. doi:10.1021/acs.iecr.8b04671.

80. Herschy, B.; Whicher, A.; Camprubi, E.; Watson, C.; Dartnell, L.; Ward, J.; Evans, J.R.G.; Lane, N. An Origin-of-Life Reactor to Simulate Alkaline Hydrothermal Vents. Journal of Molecular Evolution 2014, 79, 213-227. doi:10.1007/s00239-014-9658-4.

81. Attwater, J.; Wochner, A.; Pinheiro, V.B.; Coulson, A.; Holliger, P. Ice as a protocellular medium for RNA replication. Nature Communications 2010, 1, 76. doi:10.1038/ncomms1076.

82. Xu, J.; Chmela, V.; Green, N.J.; Russell, D.A.; Janicki, M.J.; Góra, R.W.; Szabla, R.; Bond, A.D.; Sutherland, J.D. Selective prebiotic formation of RNA pyrimidine and DNA purine nucleosides. Nature 2020, 582, 60-66. doi:10.1038/s41586-020-2330-9.

83. Xue, M.; Black, R.A.; Cornell, C.E.; Drobny, G.P.; Keller, S.L. A Step toward Molecular Evolution of RNA: Ribose Binds to Prebiotic Fatty Acid Membranes, and Nucleosides Bind Better than Individual Bases Do. ChemBioChem 2020. doi:10.1002/cbic.202000260.

84. Becker, S.; Feldmann, J.; Wiedemann, S.; Okamura, H.; Schneider, C.; Iwan, K.; Crisp, A.; Rossa, M.; Amatov, T.; Carell, T. Unified prebiotically plausible synthesis of pyrimidine and purine RNA ribonucleotides. Science 2019, 366, 76-82. doi:10.1126/science.aax2747.

85. Ranjan, S.; Todd, Z.R.; Rimmer, P.B.; Sasselov, D.D.; Babbin, A.R. Nitrogen Oxide Concentrations in Natural Waters on Early Earth. Geochemistry, Geophysics, Geosystems 2019, 20, 2021-2039. doi:10.1029/2018gc008082. 
86. Lyons, T.W.; Reinhard, C.T.; Planavsky, N.J. The rise of oxygen in Earth's early ocean and atmosphere. Nature 2014, 506, 307. doi:10.1038/nature13068.

87. Walker, S.I.; Packard, N.; Cody, G.D. Re-conceptualizing the origins of life. Philosophical Transactions of the Royal Society A 2017, 375, 20160337. doi:10.1098/rsta.2016.0337.

88. Lopez, A.; Fiore, M. Investigating Prebiotic Protocells for A Comprehensive Understanding of the Origins of Life: A Prebiotic Systems Chemistry Perspective. Life 2019, 9, 49. doi:10.3390/life9020049.

89. Albert, B., G.B.; Damidot, D. Water chemical potential: A key parameter to determine the thermodynamic stability of some hydrated cement phases in concrete? Cement and Concrete Research 2006, 36, 783-790. doi:doi:10.1016/j.cemconres.2005.12.016.

90. Poch, O.; Jaber, M.; Stalport, F.; Nowak, S.; Georgelin, T.; Lambert, J.F.; Szopa, C.; Coll, P. Effect of Nontronite Smectite Clay on the Chemical Evolution of Several Organic Molecules under Simulated Martian Surface Ultraviolet Radiation Conditions. Astrobiology 2015, 15, 221-237. doi:10.1089/ast.2014.1230.

91. Saïagh, K.; Cloix, M.; Fray, N.; Cottin, H. VUV and mid-UV photoabsorption cross sections of thin films of adenine: Application on its photochemistry in the solar system. Planetary and Space Science, 90, 90-99. doi:http://dx.doi.org/10.1016/j.pss.2013.11.002.

92. Williamson, C.E. Effects of UV radiation on freshwater ecosystems. International Journal of Environmental Studies 1996, 51, 245-256. doi:10.1080/00207239608711083. 